\title{
STOCKHOLM NATURAL RADIOCARBON MEASUREMENTS VII
}

\author{
LARS G. ENGSTRAND
}

Radioactive Dating Laboratory, Frescati, Stockholm 50, Sweden

This paper is a direct continuation of Stockholm VI. The bone samples analyzed and published in this list are treated according to a method, modified (Sellstedt, Engstrand and Gejvall, 1966) and described by Berger, Horney and Libby (1964).

\section{ACKNOWLEDGMENTS}

I am indebted to all the staff at the laboratory and especially to Mrs. Ingrid Almstedt for her excellent handling of the routine work; Mr. Sandor Vaczy for good electronic and chemical service, and keeping the equipment in continuous operation. I also want to thank Mrs. BrittMarie Lindkvist and Miss Kristina Dietmann for their secretarial work.

\section{SAMPLE DESCRIPTIONS}

I. GEOLOGIC SAMPLES

Dry fir series

$$
\text { A. Sweden }
$$

Continued from Stockholm II and VI. Coll. and subm. by Jan Lundqvist, Geol. Survey of Sweden, Stockholm.

\section{St-1579. Ö. Bunneran 64:I}

From $63^{\circ} 12^{\prime} \mathrm{N}$ Lat, $12^{\circ} 34^{\prime} \mathrm{E}$ Long, alt $810 \mathrm{~m}$.

St-1577. Tivoli 64:II

From $63^{\circ} 11^{\prime} \mathrm{N}$ Lat, $12^{\circ} 24^{\prime} \mathrm{E}$ Long, alt $800 \mathrm{~m}$.

\section{St-1920. Glensvalen}

From $63^{\circ} 03^{\prime} \mathrm{N}$ Lat, $13^{\circ} 39^{\prime} \mathrm{E}$ Long, alt $810 \mathrm{~m}$.

\section{St-1929. Båktjenjaure}

From $63^{\circ} 58^{\prime} \mathrm{N}$ Lat, $13^{\circ} 40^{\prime} \mathrm{E}$ Long, alt $700 \mathrm{~m}$.

\section{St-1642. Kärrnäset}

High-molecular, greasy organic material found deep in glaciofluvial esker at Kärrnäset $\left(63^{\circ} 57^{\prime} \mathrm{N}\right.$ Lat, $15^{\circ} 13^{\prime} \mathrm{E}$ Long), Jämtland. Subm. by Jan Lundqvist.

\section{Jämtland peat series}

Dating of peat and pollen-analytical levels in county of Jämtland. Samples indicated with "T" are only dried before preparation. The

$$
\begin{aligned}
& 8295 \pm 100 \\
& 6345 \text { B.C. }
\end{aligned}
$$

$4685 \pm 75$ 2735 B.c.

$3385 \pm 70$ 1435 B.C.

$6310 \pm 90$ 4360 в.c.

$$
295 \pm 65
$$


others are pretreated with $2 \% \mathrm{NaOH}$ solution and $\mathrm{HCl}$ solution. Coll. and subm. by Jan Lundqvist.

\section{Fånnekölen ( $61^{\circ} 47^{\prime}$ N Lat, $13 \circ 29^{\prime}$ E Long $)$}

St-1643. Fånnekölen 1

20 to $25 \mathrm{~cm}$ below surface. Picea max., Pinus min., von Post's level a.

St-1645. Fånnekölen 3

40 to $45 \mathrm{~cm}$ below surface.

St-1644. Fånnekölen 2 level $\mathrm{b}$.
$1315 \pm 65$

A.D. 635

$5945 \pm 80$

3995 B.C.

\section{St-1662. Fånnekölen 5}

$8500 \pm 110$

90 to $95 \mathrm{~cm}$ below surface. Betula max.

6550 в.C.

St-1646. Fånnekölen 4

$7605 \pm 90$

115 to $120 \mathrm{~cm}$ below surface. Beginning of peat growth.

\section{Tönningfloarna $\left(62^{\circ} 13^{\prime} \mathrm{N}\right.$ Lat, $15^{\circ} 07^{\prime}$ E Long $)$, Jämtland}

\section{St-1670. Tönningfloarna 1}

$50 \mathrm{~cm}$ below surface. Possibly von Post's level b.

St-1671. Tönningfloarna 2

$110 \mathrm{~cm}$ below surface.

\section{St-1672. Tönningfloarna 3}

$125 \mathrm{~cm}$ below surface. No. 2 or 3 represents $P_{0}$.

St-1673. Tönningfloarna 4

$360 \mathrm{~cm}$ below surface. Probably $\mathrm{A}_{0}$, possibly $\mathrm{C}_{\mathrm{o}}$ level.

St-1674. Tönningfloarna 5

$375 \mathrm{~cm}$ below surface. Beginning of ooze accumulation.
$1865 \pm 70$

A.D. 85

$3905 \pm 90$

1955 B.c.

$4360 \pm 80$

2410 B.C.

$7125 \pm 160$

5175 B.C.

$8120 \pm 110$

6170 B.c.

Malmfloten $\left(6^{\circ}{ }^{06}{ }^{\prime}\right.$ N Lat, $13^{\circ} 22^{\prime}$ E Long)

St-1758. Malmfloten 1

$35 \mathrm{~cm}$ below surface. Von Post's level b. 
St-1759. Malmfloten 2

$75 \mathrm{~cm}$ below surface. Picea max.

St-1760. Malmfloten 3

$170 \mathrm{~cm}$ below surface. Possibly $P_{0}$.

St-1761. Malmfloten 4

$205 \mathrm{~cm}$ below surface. Possibly $P_{0}$.

\section{St-1762. Malmfloten 5}

$280 \mathrm{~cm}$ below surface. Von Post's level d.

\section{St-1764. Malmfloten 6}

\section{St-1764 B. Malmfloten 6}

$340 \mathrm{~cm}$ below surface. Beginning of peat growth. St-1764 B pretreated with $2 \% \mathrm{NaOH}$ solution. St-1764 and St-1764 B diluted with "dead" $\mathrm{CO}_{2}$.

\section{Sjöarmyren (61 ${ }^{\circ} 46^{\prime} \mathrm{N}$ Lat, $14^{\circ} 1^{\prime}$ E Long)}

St-1765. Sjöarmyren 1

$$
<\mathbf{2 5 0}
$$

St-1765 B. Sjöarmyren 1

$$
<250
$$

$40 \mathrm{~cm}$ below surface. Von Post's level b. St-1765 B pretreated with $2 \% \mathrm{NaOH}$ solution.

\section{St-1767 B. Sjöarmyren 2}

$1535 \pm 75$

$65 \mathrm{~cm}$ below surface. Picea max. Pretreated with $2 \% \mathrm{NaOH}$ solution.

St-1766. Sjöarmyren 3

130 B.C.

St-1766 B. Sjöarmyren 3

$2130 \pm 100$

180 B.C.

$90 \mathrm{~cm}$ below surface. $\mathrm{P}_{\mathrm{o}}$. St-1766 B pretreated with $2 \% \mathrm{NaOH}$ solution.

St-1768. Sjöarmyren 4

$8095 \pm 245$

$285 \mathrm{~cm}$ below surface. Beginning of peat growth.

S. Mjölstötmyren ( $62^{\circ} 13^{\prime} \mathrm{N}$ Lat, $12^{\circ} 56^{\prime}$ E Long)

St-1769. S. Mjölstötmyren 1

$765 \pm 80$ 
St-1769 B. S. Mjölstötmyren 1

$35 \mathrm{~cm}$ below surface. Von Post's level b. St-1769 B pretreated with $2 \% \mathrm{NaOH}$ solution.

\section{St-1770. S. Mjölstötmyren 2}

St-1770 B.

S. Mjölstötmyren 2

$65 \mathrm{~cm}$ below surface. $\mathrm{P}_{\mathrm{o}}$. St-1770 B pretreated with $2 \% \mathrm{NaOH}$ solution.

\section{St-1771. S. Mjölstötmyren 3}

St-1771 B. S. Mjölstötmyren 3

$230 \mathrm{~cm}$ below surface. Betula max. St-1771 B pretreated with $2 \%$ $\mathrm{NaOH}$ solution, then diluted with "dead" $\mathrm{CO}_{2}$.

\section{St-1774. S. Mjölstötmyren 4 T}

\section{St-1775. S. Mjölstötmyren 4}

$355 \mathrm{~cm}$ below surface. $A_{0}$.

\section{St-1772. S. Mjölstötmyren 5}

$360 \mathrm{~cm}$ below surface. Beginning of peat growth.

Funäsmyren $\left(62^{\circ} 35^{\prime} \mathrm{N}\right.$ Lat, $12^{\circ} 33^{\prime}$ E Long)

\section{St-1905. Funäsmyren $1 \mathrm{~T}$}

\section{St-1982. Funäsmyren 1}

$65 \mathrm{~cm}$ below surface. $P_{0}$.

\section{St-1906. Funäsmyren 2 T}

St-1983. Funäsmyren 2

$80 \mathrm{~cm}$ below surface. $P_{0}$.

\section{St-1907. Funäsmyren 3 T}

St-1984. Funäsmyren 3

$100 \mathrm{~cm}$ below surface. Betula max.
$2460 \pm 60$

510 B.c.

$2605 \pm 90$

655 B.c.

$5845 \pm 75$

3895 B.C.

$6575 \pm 410$

4625 B.c.
$9180 \pm 200$ 7230 B.C.

$9510 \pm 210$ 7560 в.c.

$9725 \pm 100$ 7775 B.c.

$$
\begin{gathered}
2015 \pm 65 \\
65 \text { B.c. } \\
1935 \pm 75 \\
\text { A.D. } 15 \\
\\
2450 \pm 100 \\
500 \text { в.c. } \\
2310 \pm \mathbf{7 0} \\
\mathbf{3 6 0} \text { в.C. } \\
\\
2910 \pm \mathbf{8 0} \\
\mathbf{9 6 0} \text { в.C. } \\
\mathbf{2 4 5 0} \pm \mathbf{6 5} \\
\mathbf{5 0 0} \text { в.C. }
\end{gathered}
$$


St-1908. Funäsmyren $4 \mathrm{~T}$

St-1985. Funäsmyren 4

$195 \mathrm{~cm}$ below surface. Beginning of peat growth.

Flatrumyren $\left(62^{\circ} 44^{\prime}\right.$ N Lat, $12^{\circ} 46^{\prime}$ E Long $)$

St-1910. Flatrumyren $1 \mathrm{~T}$

St-1986. Flatrumyren 1

$65 \mathrm{~cm}$ below surface. $\mathrm{P}_{\mathrm{o}}$.

\section{St-1911. Flatrumyren 2 T}

St-1987. Flatrumyren 2

$160 \mathrm{~cm}$ below surface. Betula max.

\section{St-1912. Flatrumyren $3 \mathrm{~T}$}

\section{St-1989. Flatrumyren 3}

$235 \mathrm{~cm}$ below surface. Beginning of peat growth.

Henvålsmyren $\left(62^{\circ} 43^{\prime} \mathbf{N}\right.$ Lat, $13^{\circ} 2^{\prime}$ E Long)

St-1913. Henvålsmyren 1 T

St-1988. Henvålsmyren 1

$25 \mathrm{~cm}$ below surface. Von Post's level b.

St-1914. Henvålsmyren 2 T

St-1990. Henvålsmyren 2

$60 \mathrm{~cm}$ below surface. $\mathrm{P}_{\mathrm{o}}$.

\section{St-1915. Henvålsmyren 3 T}

St-1991. Henvålsmyren 3

$90 \mathrm{~cm}$ below surface. Betula max.

\section{St-1917. Henvålsmyren 4 T}

St-1992. Henvålsmyren 4

$110 \mathrm{~cm}$ below surface. Beginning of peat growth.
$7670 \pm 110$ 5720 в.C.

$7320 \pm 100$ 5370 в.c.

$$
\begin{gathered}
2630 \pm 75 \\
680 \text { в.c. } \\
2440 \pm 70 \\
490 \text { в.c. } \\
\\
4360 \pm 80 \\
2410 \text { B.c. } \\
4375 \pm 110 \\
2425 \text { B.c. }
\end{gathered}
$$

$7105 \pm 105$

5155 в.c.

$7260 \pm 135$

5310 B.c.

$3400 \pm 80$

1450 B.c.

$2450 \pm 75$

500 B.C.

$6380 \pm 100$

4430 в.C.

$5830 \pm 80$

3880 B.C.

$8160 \pm 110$

6210 B.C.

$8205 \pm 170$

6255 B.C. 
Vattenfloen $\left(62^{\circ} 21^{\prime} \mathrm{N}\right.$ Lat, $12^{\circ} 41^{\prime}$ E Long $)$

St-1933. Vattenfloen 1 T

St-1993. Vattenfloen 1

$40 \mathrm{~cm}$ below surface. $P_{0}$.

St-1934. Vattenfloen 2 T

$135 \mathrm{~cm}$ below surface. Betula max.

St-1935. Vattenfloen 3 T

St-1994. Vattenfloen 3

$195 \mathrm{~cm}$ below surface. $A_{0}$.

St-1936. Vattenfloen $4 T$

St-1995. Vattenfloen 4

$200 \mathrm{~cm}$ below surface. Beginning of peat growth.
$1865 \pm 70$

A.D. 85

$1985 \pm 70$

35 B.c.

$5285 \pm 110$

3335 в.c.

$7690 \pm 100$

5740 в.C.

$8010 \pm 100$

6060 B.C.

$8075 \pm 100$

6125 B.C.

$8275 \pm 100$ 6325 B.c.

Stockbergsmyren $\left(62^{\circ} 44^{\prime} \mathrm{N}\right.$ Lat, $14^{\circ} 32^{\prime}$ E Long $)$

St-1943. Stockbergsmyren $1 \mathrm{~T}$

St-2008. Stockbergsmyren 1

$35 \mathrm{~cm}$ below surface. Von Post's level a.

St-1937. Stockbergsmyren 2 T

$85 \mathrm{~cm}$ below surface $P_{0}$.

St-1938. Stockbergsmyren 3 T

$170 \mathrm{~cm}$ below surface. Von Post's level d(?). Betula max

St-1939. Stockbergsmyren $4 \mathrm{~T}$

St-2001. Stockbergsmyren 4

$220 \mathrm{~cm}$ below surface. Betula increase/Pinus decrease. Beginning of QM curve.

St-1940. Stockbergsmyren 5 T

St-2000. Stockbergsmyren 5

$285 \mathrm{~cm}$ below surface. $\mathrm{A}_{0}$.
$430 \pm 70$

A.D. 1520

A.D. 1505

$445 \pm 65$

$2440 \pm 75$

490 B.C.

$4830 \pm 80$

2880 B.C.
$6310 \pm 100$

4360 B.C.

$6255 \pm 90$

4305 в.c.

$8365 \pm 100$

6415 B.C.

$8595 \pm 180$

6645 B.C. 
St-1941. Stockbergsmyren 6 T

$8340 \pm 120$

6390 B.C.

St-2002. Stockbergsmyren 6

$9055 \pm 180$

$315 \mathrm{~cm}$ below surface. Mountain heath vegetation ends.

St-1942. Stockbergsmyren $7 \mathrm{~T}$

$8835 \pm 105$

6885 B.C.

$9280 \pm 170$

St-2009. Stockbergsmyren 7

7330 B.C.

$340 \mathrm{~cm}$ below surface. Beginning of peat growth.

Docksmyren $\left(62^{\circ} 56^{\prime}\right.$ N Lat, $15^{\circ} 44^{\prime}$ E Long $)$

St-1944. Docksmyren 1 T

$1035 \pm 70$

A.D. 915

St-2035. Docksmyren 1

$1000 \pm 70$

$65 \mathrm{~cm}$ below surface. Von Post's level a.

St-1945. Docksmyren 2 T

St-2026. Docksmyren 2

$150 \mathrm{~cm}$ below surface. $P_{0}$.

St-1946. Docksmyren 3 T

St-2036. Docksmyren 3

$315 \mathrm{~cm}$ below surface. Von Post's level d.

St-1948. Docksmyren $4 \mathrm{~T}$

St-2027. Docksmyren 4

$330 \mathrm{~cm}$ below surface. Beginning of QM curve.

St-1949. Docksmyren 5 T

St-2037. Docksmyren 5

A.D. 950

$2730 \pm 75$

1780 B.C.

$2410 \pm 75$

460 B.C.

$5930 \pm 80$

3980 B.C.

$6170 \pm 80$

4220 в.C.

$6540 \pm 90$

4590 в.c.

$5845 \pm 80$

3895 B.C.

$7200 \pm 100$

5250 в.C.

$7775 \pm 185$

5825 B.C.

$350 \mathrm{~cm}$ below surface. Beginning of peat growth.

Varggransmyren $\left(62^{\circ} \mathbf{2 8}^{\prime} \mathrm{N}\right.$ Lat, $1^{\circ} \mathrm{00}^{\prime}$ E Long $)$

St-1955. Varggransmyren $1 \mathrm{~T}$

$<\mathbf{2 5 0}$

St-2041. Varggransmyren 1

$<\mathbf{2 5 0}$

$30 \mathrm{~cm}$ below surface. Picea max., von Post's level a? 
St-1956. Varggransmyren 2 T

St-2051. Varggransmyren 2

$70 \mathrm{~cm}$ below surface. $\mathrm{P}_{0}$.

St-2126. Varggransmyren 4

$125 \mathrm{~cm}$ below surface.

St-1957. Varggransmyren 3 T

$130 \mathrm{~cm}$ below surface. Beginning of peat growth.

Myssjö Storflo ( $63^{\circ} 5^{\prime}$ N Lat, $1^{\circ}$ 08 $^{\prime}$ E Long)

St-1963. Myssjö Storflo 1 T

St-2053. Myssjö Storflo 1

$25 \mathrm{~cm}$ below surface. Von Post's level b.

St-1964. Myssjö Storflo 2 T

St-2054. Myssjö Storflo 2

$60 \mathrm{~cm}$ below surface. $P_{0}$.

St-2058. Myssjö Storflo 7

$65 \mathrm{~cm}$ below surface.

St-2060. Myssjö Storflo 8

$80 \mathrm{~cm}$ below surface.

St-1965. Myssjö Storflo 3 T

St-2055. Myssjö Storflo 3

$95 \mathrm{~cm}$ below surface. Zone boundary III/IV.

St-2061. Myssjö Storflo 9

$110 \mathrm{~cm}$ below surface.

St-1966. Myssjö Storflo 4 T

$120 \mathrm{~cm}$ below surface. Picea max.

St-2062. Myssjö Storflo 10

$130 \mathrm{~cm}$ below surface.

$$
\begin{gathered}
2120 \pm 70 \\
170 \text { B.c. } \\
2175 \pm 75 \\
225 \text { B.c. } \\
\\
4515 \pm 80 \\
2565 \text { B.c. }
\end{gathered}
$$

$5010 \pm 120$

3060 B.c.

$<\mathbf{2 5 0}$

$1635 \pm 75$ A.D. 315

$1725 \pm 70$ A.D. 225

$2355 \pm 70$

405 B.C.

$2795 \pm 70$

845 B.c.

$3500 \pm 70$

1550 в.c.

$3425 \pm 100$

1475 B.c.

$4310 \pm 70$

2360 B.C.

$2505 \pm 80$

555 в.c.

$3920 \pm 75$

1970 B.C. 
$5330 \pm 80$

St-1967. Myssjö Storflo 5 T

3380 B.C.

St-2049. Myssjö Storflo 5

$5865 \pm 90$

$155 \mathrm{~cm}$ below surface. Von Post's level d.

3915 B.c.

St-1968. Myssjö Storflo 6 T

$7710 \pm 100$

5760 B.C.

$8010 \pm 100$

St-2056. Myssjö Storflo 6

6060 B.C.

$190 \mathrm{~cm}$ below surface. Beginning of peat growth.

Bjurälvsmossen $\left(64^{\circ}{55^{\prime}}^{N}\right.$ Lat, $1^{\circ} 0^{\prime}$ E Long $)$

St-2097. Bjurälvsmossen $1 \mathrm{~T}$

$2330 \pm 75$

380 B.C.

$2320 \pm 75$

370 B.c.

St-2090. Bjurälvsmossen 1

$4790 \pm 75$

$90 \mathrm{~cm}$ below surface. $P_{0}$.

St-2091. Bjurälvsmossen 2

2840 B.C.

$145 \mathrm{~cm}$ below surface. Change from Alnus to Betula domain. Beginning of peat growth.

Laxsjömyren $\left(63^{\circ} 49^{\prime}\right.$ N Lat, $14^{\circ} 51^{\prime}$ E Long $)$

St-2098. Laxsjömyren 1

$2495 \pm 75$

545 в.c.

$75 \mathrm{~cm}$ below surface $P_{o}$.

St-2099. Laxsjömyren 2

$4950 \pm 80$

3000 B.c.

$110 \mathrm{~cm}$ below surface. Betula max.

St-2101. Laxsjömyren 3

$7340 \pm 100$

5390 в.c.

$220 \mathrm{~cm}$ below surface. Beginning of peat growth.

Hallvikenmyren ( $63^{\circ} 44^{\prime} \mathbf{N}$ Lat, $15^{\circ} 33^{\prime}$ E Long)

St-2076. Hallviken 1 T

$1305 \pm 70$

A.D. 645

$1240 \pm 65$

St-2074. Hallviken 1

A.D. 710

$65 \mathrm{~cm}$ below surface. Picea max., von Post's level b.

St-2071. Hallviken 2 T

$2485 \pm 90$

535 в.c.

St-2075. Hallviken 2

$2615 \pm 100$

665 B.C. 
St-2079. Hallviken 3

$5410 \pm 130$

$265 \mathrm{~cm}$ below surface. Betula max., von Post's level d?

St-2070. Hallviken 4 T

$6650 \pm 80$

4700 в.C.

St-2081. Hallviken 4

$7320 \pm 145$

$300 \mathrm{~cm}$ below surface. $\mathrm{U}_{\mathrm{o}}$ and $\mathrm{C}_{\mathrm{o}}$.

5370 B.c.

St-2073. Hallviken 5 T

$7420 \pm 150$

5470 в.c.

St-2086. Hallviken 5

$7920 \pm 160$

5970 в.c.

$340 \mathrm{~cm}$ below surface. Betula max., von Post's level d?

St-2072. Hallviken 6 T

$8665 \pm 230$

6715 B.C.

St-2080. Hallviken 6

$8275 \pm 140$

$395 \mathrm{~cm}$ below surface. $A_{0}$.

6325 в.C.

\section{St-2083. Hallviken 7}

$9860 \pm 115$

7910 B.C.

$405 \mathrm{~cm}$ below surface. Beginning of organic sedimentation.

Predikmyren (64 ${ }^{\circ} 58^{\prime}$ N Lat, 14 $^{\circ}$ 08' $^{\prime}$ E Long)

St-2096. Predikmyren $1 \mathrm{~T}$

$\mathbf{5 0} \pm \mathbf{7 0}$

St-2094. Predikmyren 1

$25 \mathrm{~cm}$ below surface. Picea rise. $\left(\mathbf{P}_{0}\right.$ ?)

$1210 \pm 65$

A.D. 740

St-2095. Predikmyren 2 T

$3240 \pm 75$

1290 B.C.

St-2092. Predikmyren 2

$2945 \pm 75$

$60 \mathrm{~cm}$ below surface. $P_{0}$.

995 B.C.

St-2093. Predikmyren 3

$6685 \pm 75$

$140 \mathrm{~cm}$ below surface. Beginning of peat growth.

4735 B.C.

Lidsjömyren $\left(64^{\circ} 19^{\prime}\right.$ N Lat, $15^{\circ} 1^{\prime}$ E Long $)$

St-2102. Lidsjömyren 1

$2395 \pm 75$

$150 \mathrm{~cm}$ below surface. $P_{0}$.

445 B.c. 
St-2103. Lidsjömyren 2

$4630 \pm 80$

$225 \mathrm{~cm}$ below surface. Betula max.

St-2104. Lidsjömyren 3

$8525 \pm 100$

$305 \mathrm{~cm}$ below surface. $A_{0}$.

6575 B.C.

\section{St-2085. Lidsjömyren 4}

$9620 \pm 180$

$315 \mathrm{~cm}$ below surface. Beginning of peat growth.

7670 B.C.

St-2082. Lidsjömyren 5 T

$8500 \pm 100$

6550 B.C.

$545 \mathrm{~cm}$ below surface. Beginning of mud sedimentation.

\section{Åflon $\left(63^{\circ} 28^{\prime} \mathbf{N}\right.$ Lat, $13^{\circ} 50^{\prime} \mathrm{E}$ Long $)$}

St-2127. Åflon 1

$2120 \pm 65$

$110 \mathrm{~cm}$ below surface. $P_{0}$.

170 B.C.

\section{St-2125. Åflon 2}

$4530 \pm 75$

$270 \mathrm{~cm}$ below surface. Betula max.

2580 B.C.

St-2129. Åflon 3

$5795 \pm 135$

$365 \mathrm{~cm}$ below surface. $\mathrm{T}_{\mathrm{o}}$.

3845 B.C.

St-2130. Åflon 4

$6725 \pm 90$

4775 B.C.

$405 \mathrm{~cm}$ below surface. Betula max.

\section{St-2131. Åflon 5}

$6760 \pm 100$

$425 \mathrm{~cm}$ below surface. $\mathrm{QM}_{0}$.

\section{The West Coast-Kattegatt series}

Peat, "pitchy soil," mud (with various amount of clay and detritus) and shell from Swedish West Coast between Marstrand and Kullen (58 to $56^{\circ} \mathrm{N}$ Lat), and Kattegatt Sea between Sweden and Denmark $\left(10.5^{\circ}\right.$ to $12.5^{\circ} \mathrm{E}$ Long). Dated in order to connect shorelines and date shore level displacement on Swedish West Coast and in Kattegatt Sea during Late Glacial and Postglacial time. Coll. and subm. by Nils-Axel Mörner, Dept. of Quaternary Geology, Univ. of Stockholm.

St-2168. B 579

$11,235 \pm 235$

Clayey mud.

9285 B.C.

St-2163. B 574:I

$11,170 \pm 240$

Mud/clay.

9220 B.C. 
St-2164. B 574:II

Mud, clayey.

St-2165. B 574:III

Mud.

St-2003. B 432

Detritus mud.

St-2006. B 437

Detritus mud.

St-2167. B 551

Mud.

St-2175. B 557

Mud.

St-2166. B 566

Muddy clay.

St-2203. B 583:I

Macoma calcarea.

St-2204. B 583:II

Balanus hameri.

St-2176. B 583:III

St-2010. B 427:II

Mud.

St-2005. B 438:II

Coarse-detritus mud.

St-2170. B 441:II

Clayey mud.

St-1707. B 1561

Mud.
$10,225 \pm 120$

8275 B.C.

$9970 \pm 160$

8020 ....

$10,400 \pm 220$

8450 B.C.

$8120 \pm 210$

6170 B.C.

$7525 \pm 180$

5575 B.C.

$9725 \pm 115$

7775 B.C.

$4210 \pm 130$

2260 B.C.

$7200 \pm 130$

5250 B.C.

$11,565 \pm 150$

9615 B.C.

$1985 \pm 115$

35 B.c.

yttja).

$10,000 \pm 330$ 8050 B.C.

$10,285 \pm 120$ 8335 B.C.

$9780 \pm 200$

7830 B.C.

$9400 \pm 450$

7450 B.C. 
St-1710. B 3

Fine (coarse) detritus mud.

St-2004. B 441 :I

Coarse detritus mud.

St-1698. B 171

Coarse detritus mud (Erioph., Phragm.).

St-1816. B 145:N+L

Black peat-"pitchy soil."

St-1814. B 145:N(R)

Dark peat.

\section{St-1817. B 143:H}

"Pitchy soil" and mud.

St-1813. B $143: H(R)$

Mud-clayey mud.

St-2169. B 501

Dy.

St-2211. B 286

Dy and shells.

\section{St-2206. B 287}

Littorina sp. and shell fragments.

St-2171. B 502

Wood and dy-peat.

St-2174. B 504

Wood and dy-peat.

St-1820. B 176:I

Peat.

St-2172. B 176:II

Brushwood peat.
$9700 \pm 120$

7750 B.C.

$9280 \pm 130$

7330 B.C.

$9155 \pm 120$ 7205 B.C.

$9435 \pm 180$ 7485 B.c.

$9280 \pm 300$ 7330 B.c.

$9130 \pm 120$ 7180 в.c.

$9230 \pm 500$ 7280 B.C.

$9280 \pm 125$ 7330 B.C.

$3255 \pm 80$ 1305 B.C.

$8735 \pm 105$ 6785 B.C.

$10,820 \pm 200$ 8870 в.C.

$9725 \pm 200$ 7775 B.C.

$7565 \pm 110$

5615 в.C.

$8010 \pm 100$

6060 B.C. 
St-2007. B 450

Coarse detritus mud, clayey.

St-2011. B 438:I

Coarse detritus mud.

St-2012. B 427:I

Black peat.

St-1818. B 268:I

"Pitchy soil."

St-2177. B 268:II

Peat.

St-1711. B 228:I

Clayey, muddy peat (bushwood peat).

St-1815. B 228:II

Peat (bushwood peat).

St-2214. B 228:III

Peat (bushwood peat).

St-1819. B 220

"Pitchy soil."

St-2173. B 552

Humus layer in sand.

St-2158. B 325

Peat.

\section{Rya series}

Clay and peat from Rya ( $57^{\circ} 33^{\prime} \mathrm{N}$ Lat, $12^{\circ} 25^{\prime} \mathrm{E}$ Long), valley of Lygnern River, Västergötland. Investigation made to date different shorelines. Coll. and subm. by G. Ljunggren-Smedman, Dept. of Quaternary Geology, Univ. of Stockholm.

St-1891. Rya 1:40

$5025 \pm 80$

Clay $40 \mathrm{~cm}$ below surface.
$7400 \pm 100$

5450 B.C.

$7565 \pm 160$

5615 B.c.

$8595 \pm 100$

6645 в.c.

$7220 \pm 150$

5270 B.c.

$6855 \pm 90$

4905 B.C.

$6520 \pm 105$

4570 в.C.

$3005 \pm 80$

1055 B.C.

$5780 \pm 95$

3830 B.C.

$6430 \pm 140$

4480 в.C.

$2100 \pm 75$

150 B.C.

$1985 \pm 70$

35 B.c. 
St-1890. Rya $1: 80$

$5680 \pm 125$

Clay $80 \mathrm{~cm}$ below surface.

3730 B.C.

St-1892. Rya 1:260

$8365 \pm 260$

6415 B.c.

Peat $260 \mathrm{~cm}$ below surface.

$8905 \pm 170$

St-1894. Rya $1: 284$

6955 в.c.

Clay $284 \mathrm{~cm}$ below surface.

Skede mosse series

Continued from Stockholm VI. Location: Skede mosse $\left(56^{\circ} 05^{\prime} \mathrm{N}\right.$ Lat, $16^{\circ} 45^{\prime}$ E Long), Gärdslösa parish, öland. Coll. and subm. by L. K. Königsson, Dept. of Quaternary Geology, Univ. of Uppsala, Uppsala.

\begin{tabular}{|c|c|c|}
\hline St-1821. & $2 G$ & $\begin{array}{l}1780 \pm 75 \\
\text { A.D. } 170\end{array}$ \\
\hline St-1822. & $6 \mathrm{G}$ & $\begin{array}{l}4530 \pm 100 \\
2580 \text { B.C. } \\
9080 \pm 145\end{array}$ \\
\hline St-1823. & $3 \mathrm{G}$ & $\begin{array}{l}7130 \text { в.c. } \\
9030 \pm 120\end{array}$ \\
\hline St-1824. & $8 \mathrm{G}$ & $\begin{array}{l}7080 \text { B.C. } \\
2935 \pm 100\end{array}$ \\
\hline St-1825. & $11 \mathrm{G}$ & $\begin{array}{l}985 \text { B.C. } \\
3520 \pm 70\end{array}$ \\
\hline St-1826. & $12 \mathrm{G}$ & $\begin{array}{c}1570 \text { B.C. } \\
3700 \pm 100\end{array}$ \\
\hline St-1827. & $16 \mathrm{G}$ & $\begin{array}{l}1750 \text { B.C. } \\
4025 \pm 100\end{array}$ \\
\hline St-1828. & $17 \mathrm{G}$ & $\begin{array}{l}2075 \text { B.C. } \\
3890 \pm 80\end{array}$ \\
\hline St-1829. & $18 \mathrm{G}$ & $\begin{array}{l}1940 \text { B.C. } \\
3855 \pm 95\end{array}$ \\
\hline St-1830. & $19 \mathrm{G}$ & $\begin{array}{c}1905 \text { B.C. } \\
8970 \pm 155\end{array}$ \\
\hline St-1832. & VIII: 1 G & $\begin{array}{l}7020 \text { в.с. } \\
9055 \pm 140\end{array}$ \\
\hline kadalen & VIII:2 G & / 105 B.c. \\
\hline
\end{tabular}


deltas. Coll. and subm. by Stig Widell, Dept. of Quaternary Geology, Univ. of Stockholm.

\section{St-2038. B.P. 11:50-160}

$9915 \pm 130$

Clay, 150 to $160 \mathrm{~cm}$ below surface.

St-1899. B.P. $11: 240-260$

7965 в.C.

Wood, 240 to $260 \mathrm{~cm}$ below surface.

\section{St-2021. B.P. 11:300-320}

$9330 \pm 110$

7380 в.C.

Clay, 300 to $320 \mathrm{~cm}$ below surface.

\section{St-1897. B.P. 4:150-160}

$10,400 \pm 120$

8450 B.C.

Wood, 150 to $160 \mathrm{~cm}$ below surface.

St-2028. B.P. 4:250-260

Clay, 250 to $260 \mathrm{~cm}$ below surface.

St-1895. B.P. 8:480-490

$3180 \pm 75$

1230 B.c.

Clay, 480 to $490 \mathrm{~cm}$ below surface.

\section{St-1896. B.P. 6:440-450}

$5055 \pm 80$ 3105 B.c.

Wood, 440 to $450 \mathrm{~cm}$ below surface.

St-1900. B.P. 1:450-460

$3460 \pm 105$

1510 B.C.

$\mathbf{3 7 5 0} \pm \mathbf{7 0}$

1800 B.C.

Wood, 450 to $460 \mathrm{~cm}$ below surface.

$4405 \pm 80$

2455 B.C.

\section{Nyhem series}

Peat covered by eolian sand from Nyhem $\left(56^{\circ} 40^{\prime} \mathrm{N}\right.$ Lat, $14^{\circ} 54^{\prime} \mathrm{E}$ Long), Halmstad, Halland. Subm. by E. Mohrén, Geol. Survey of Sweden. Coll. from soil surface $+7 \mathrm{~m}$ above sealevel; on fine marine sand on flat plain below Littorina shoreline $(+12 \mathrm{~m})$, in remains of Phragmites peat ca. $0.75 \mathrm{~m}$ thick. Marine sand and peat have been pollen-analyzed.

\section{St-1748. 48-51}

$2450 \pm 65$

Fen peat with alder, willow etc.

500 B.C.

St-1749. 86-91

$2990 \pm 120$

1040 B.C. sea.

Lowest of the peat, representing isolation of lagoon swamps from

\section{St-1684. Handöl}

$3710 \pm 80$

1760 B.C.

Peat from Handöl (63 N Lat, $24^{\circ}$ E Long), Jämtland. Subm. by C. G. Wenner, Dept. of Quaternary Geology, Univ. of Stockholm. 


\section{Slåboda mosse series}

Sphagnum peat from Slåboda (590 $09^{\prime} \mathrm{N}$ Lat, $18^{\circ} 00^{\prime} \mathrm{E}$ Long), Västerhaninge, Södermanland. Coll. by Carl Larsson; subm. by Hans Möller, Geol. Survey of Sweden, Stockholm.

\section{St-2067. Slåboda 2}

$1430 \pm 75$

165 to $170 \mathrm{~cm}$ below surface.

\section{St-2069. Slåboda 3}

155 to $160 \mathrm{~cm}$ below surface.

$$
1145 \pm 65
$$

\section{Tornbergasjön series}

Clayey mud from moor at lake Tornbergasjön $\left(59^{\circ} 08^{\prime} \mathrm{N}\right.$ Lat, $18^{\circ}$ 05' E Long), Västerhaninge, Södermanland. Coll. by Carl Larsson; subm. by Hans Möller.

\section{St-2040. Tornbergasjön 4}

$9155 \pm 180$

475 to $480 \mathrm{~cm}$ below surface. 7205 B.C.

\section{St-2039. Tornbergasjön 5}

$9005 \pm 225$

480 to $490 \mathrm{~cm}$ below surface.

\section{Blekinge vegetational history series}

To get an absolute chronology for Late Quaternary time in eastern Blekinge several series of gyttja and peat samples were collected and dated. All samples are derived from pollen-analyzed sections in lakes or bogs. Results are discussed more comprehensively by Berglund (1966 a, b), the Hallarums mosse series also by Berglund (1964). Coll. and subm. by B. E. Berglund, Dept. of Quaternary Geology, Univ. of Lund, Lund.

\section{Lösensjön}

Gyttja samples from section in the lake of Lösensjön $\left(56^{\circ} 13^{\prime} \mathrm{N}\right.$ Lat, $15^{\circ} 45^{\prime}$ E Long), Lösen parish, Blekinge. Samples date Late-Glacial pollen zones. They were cut from core (diam $36 \mathrm{~mm}$ ) taken by Livingstone sampler. Depth given is that below water level (depth of water $270 \mathrm{~cm}$ ).

\section{St-1421. Lösensjön 4}

$10,520 \pm 170$

Muddy clay with mosses, 684 to $688 \mathrm{~cm}$. Dates end of Younger Dryas period, but value is higher than expected.

\section{St-1422. Lösensjön 10}

$$
10,400 \pm 250
$$
period.

Muddy clay, 710 to $1716 \mathrm{~cm}$. Dates beginning of Younger Dryas 
St-1683. Lösensjön 12-13

$$
11,300 \pm 360
$$

9350 B.c.

Clay gyttja, 724 to $740 \mathrm{~cm}$. Dates medium of Alleröd period.

\section{St-1423. Lösensjön 16}

$11,740 \pm 170$ period.

Clay gyttja with mosses, 751 to $761 \mathrm{~cm}$. Dates beginning of Alleröd

\section{Igelsjön}

Clayey algae gyttja from section in the lake of Igelsjön $\left(56^{\circ} 13^{\prime} \mathrm{N}\right.$ Lat, 15 42' E Long), Augerum parish, Blekinge. Dates end of Alleröd period. It is cut from core (diam $36 \mathrm{~mm}$ ) taken by Livingstone piston sampler. Depth below water level 1015 to $1021 \mathrm{~cm}$ (depth of water 505 $\mathrm{cm})$.

\section{St-1420. Igelsjön 34}

$$
\begin{aligned}
& 10,850 \pm 220 \\
& 8900 \text { в.C. }
\end{aligned}
$$

\section{Slättmossen}

Clay gyttja from section in the bog Slättmossen $\left(56^{\circ} 09^{\prime} \mathrm{N}\right.$ Lat, $15^{\circ} 51^{\prime} \mathrm{E}$ Long), Jämjö parish, Blekinge. Samples were cut out from core (diam $60 \mathrm{~mm}$ ) taken by Livingstone piston sampler. Date the transition Younger Dryas/Pre-Boreal time.

\section{St-1778. Slättmossen 1}

$10,170 \pm 160$

Clay gyttja, 346 to $350 \mathrm{~cm}$ below bog surface.

\section{St-1847. Slättmossen 2}

$9700 \pm 150$

Clay gyttja, 362 to $366 \mathrm{~cm}$ below bog surface.

\section{Hallarums mosse}

Gyttja samples from pollen- and diatom-bearing section in the bog

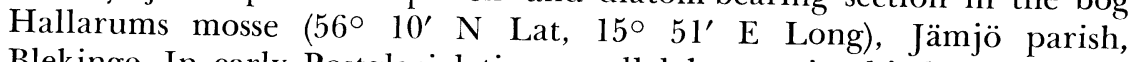
Blekinge. In early Postglacial time small lake was in this basin, but in Early Atlantic time Littorina Sea transgressed. At end of Sub-Boreal time basin was again isolated. All samples were cut out from core (diam 60 $\mathrm{mm}$ ) taken by piston sampler (type Borro). Depth given is that below surface of bog. Age figures of marine samples must be reduced. Correction has been proposed to be ca. $400 \mathrm{yr}$ (Berglund, 1964).

\section{St-1324. Hallarums mosse 15}

$4585 \pm 70$

Marine, slightly clayey fine detritus gyttja, 221 to 224 cm5 B.c. Sub-Boreal time immediately below isolation level. 
$\mathbf{5 0 4 0} \pm \mathbf{8 5}$

St-1325. Hallarums mosse 21

3090 B.C.

Marine fine detritus gyttja, 244 to $248 \mathrm{~cm}$. Early Sub-Boreal time.

\section{St-1326. Hallarums mosse 25}

$5665 \pm 85$

3715 B.c.

Marine, clayey fine detritus gyttja, 260 to $264 \mathrm{~cm}$. Dates beginning of Early Sub-Boreal period.

\section{St-1327. Hallarums mosse 30}

$5945 \pm 75$

3995 B.C. period.

Marine fine detritus gyttja, 280 to $284 \mathrm{~cm}$. Dates end of Late Atlantic

\section{St-1328. Hallarums mosse 34}

$6220 \pm 85$

4270 B.c.

Marine fine detritus gyttja, 296 to $300 \mathrm{~cm}$. Dates beginning of Late Atlantic period.

\section{St-1330. Hallarums mosse 37}

Marine fine detritus gyttja, 308 to $312 \mathrm{~cm}$. Dates very end of Early Atlantic period.

\section{St-1331. Hallarums mosse 41}

$6950 \pm 90$

5000 в.c.

Brackish fine detritus gyttja, 324 to $328 \mathrm{~cm}$. Dates middle of Early Atlantic period and first Littorina transgression in this basin.

\section{St-1332. Hallarums mosse 44}

$7105 \pm 90$

Lacustrine coarse detritus gyttja, 936 to $940 \mathrm{~cm}$. From midlle of Early Atlantic period immediately before transgression of Littorina Sea in this basin.

\section{St-1471. Hallarums mosse 50}

$7710 \pm 150$

5760 B.C.

Lacustrine, slightly clayey fine detritus gyttja, 360 to $364 \mathrm{~cm}$. Dates transition Boreal/Atlantic.

\section{St-1333. Hallarumas mosse 56}

$8140 \pm 90$

Lacustrine, slightly clayey fine detritus gyttja, 384 to $388 \mathrm{~cm}$. Dates middle of Late Boreal period.

\section{St-1472. Hallarums mosse 66}

$8665 \pm 115$

6715 B.C.

Lacustrine, clayey fine detritus gyttja, 424 to $428 \mathrm{~cm}$. Dates very beginning of Late Boreal period.

\section{St-1334. Hallarums mosse 70}

$8835 \pm 170$

Lacustrine, clayey fine detritus gyttja, 440 to $444 \mathrm{~cm}$. Dates very end of Early Boreal period. 


\section{St-1335. Hallarums mosse 83}

Lacustrine, slightly clayey fine detritus gyttja, 492 to $496 \mathrm{~cm}$. Dates very beginning Early Boreal period.

\section{St-1679. Hallarums mosse 84}

$9380 \pm 120$

7430 B.C.

Lacustrine, slightly clayey fine detritus gyttja, 496 to $500 \mathrm{~cm}$. Dates the transition Pre-Boreal/Boreal.

\section{St-1680. Hallarums mosse 88-89}

$9780 \pm 115$

Lacustrine, slightly clayey fine detritus gyttja, 512 to $520 \mathrm{~cm}$. Dates middle of Pre-Boreal period.

\section{St-1336. Hallarums mosse 95}

$$
10,000 \pm 170
$$

8050 B.C.

Lacustrine, clayey algae gyttja, 540 to $544 \mathrm{~cm}$. Dates the transition Younger Dryas/Pre-Boreal.

\section{St-1337. Hallarums mosse 97-98}

$$
10,170 \pm 230
$$

Lacustrine, clayey algae gyttja and muddy clay, 548 to $556 \mathrm{~cm}$. Dates the transition Younger Dryas/Pre-Boreal.

\section{Bäcks mosse}

Peat samples from section in the raised bog Bäcks mosse on island of Aspö (56 $06^{\circ}$ N Lat, $15^{\circ} 32^{\prime}$ Long), Blekinge. Samples are taken in a dug wall. Depth given is that below surfaces of bog.

\section{St-1289. Bäcks mosse 125}

$$
2715 \pm 80
$$

Highly humified Sphagnum peat, 30 to $34 \mathrm{~cm}$. Dates end of SubBoreal time.

\section{St-1293. Bäcks mosse 131}

$4405 \pm 80$

Highly humified Sphagnum peat, 52 to $56 \mathrm{~cm}$. Dates middle of Early Sub-Boreal time.

\section{St-1682. Bäcks mosse 135}

Highly humified Sphagnum peat, 68 to $72 \mathrm{~cm}$. Dates beginning of Early Sub-Boreal time.

\section{St-1294. Bäcks mosse 138}

$$
\mathbf{5 5 7 0} \pm \mathbf{8 5}
$$

Highly humifed sp Atlantic time.

\section{Inlängan}

Gyttja and peat from section in small bog on island of Inlängan, (56 $06^{\circ}$ N Lat, $15^{\circ} 46^{\prime}$ E Long), Torhamn parish, Blekinge. Samples 
date land occupation phase during Neolithic time in outer-most archipelago of Blekinge. They also date isolation of basin from Littorina Sea. All samples were cut out from core taken by simple tube sampler. Depth given is that below surface of bog.

\section{St-1404. Inlängan 54}

$2705 \pm 75$

Swamp peat, containing gyttja and drift, 70 to $74 \mathrm{~cm}$. Late SubBoreal time.

\section{St-1405. Inlängan 56}

$3145 \pm 65$

Lacustrine coarse detritus gyttja, 80 to $85 \mathrm{~cm}$. Dates beginning of Late Sub-Boreal time.

\section{St-1406. Inlängan 59}

$3545 \pm 65$

Brackish, slightly clayey fine detritus gyttja, 95 to $100 \mathrm{~cm}$. Dates a land occupation phase, slightly before isolation of basin at the transition Early/Late Sub-Boreal.

\section{St-1407. Inlängan 62}

$4170 \pm 75$

2220 B.C.

Brackish, slightly clayey fine detritus gyttja, 110 to $114 \mathrm{~cm}$. Early Sub-Boreal time.

\section{Bredasund}

Brackish, clayey gyttja from section in bay of the Baltic, $\left(56^{\circ} 09^{\prime} \mathrm{N}\right.$ Lat, $15^{\circ} 20^{\prime}$ E Long), Listerby parish, Blekinge. Sample is cut from core ( $60 \mathrm{~mm}$ diam) taken by Livingstone sampler. Depth below water level is 127 to $130 \mathrm{~cm}$ (depth of water $90 \mathrm{~cm}$ ). Sample dates distinct increase of human influence in vegetation in beginning of Late Sub-Atlantic time.

\section{St-1777. Bredasund}

$$
980 \pm 165
$$

\section{Utlängan}

Brackish, clayey and silty gyttja from section in the fen Marbäcken on island of Utlängan, ( $56^{\circ} 01^{\prime} \mathrm{N}$ Lat, $\left.15^{\circ} 48^{\prime} \mathrm{E} \mathrm{Long}\right)$, Torhamn parish, Blekinge. Sample is taken 43 to $47 \mathrm{~cm}$ below surface in a dug wall. Dates foundation of the farmer village on Utlängan situated in outermost archipelago of Blekinge.

\section{St-1288. Utlängan}

$$
385 \pm 70
$$

\section{Gotland series I}

Samples from the island Gotland in the Baltic Sea. Coll. and subm. by G. Lundqvist, Geol. Survey of Sweden. 
Ancylus shoreline and Littorina shoreline are two big transgression shorelines developed by the Baltic. They have been of greatest importance for dating of the geologic and archaeologic (see II, A, Archaeological samples, Sweden, Gotland series II) phases in late Quaternary time of Sweden. $\mathrm{C}^{14}$ datings of many samples of peat and wood collected under these transgression shorelines have been performed (Lundqvist, 1965).

\section{Mällingsmyr}

Peat and clay from Mällingsmyr (57 $15^{\prime} \mathrm{N}$ Lat, $18^{\circ} 13^{\prime} \mathrm{E}$ Long). Made in order to determine beginning of Littorina Sea.

St-1589. Mällingsmyr 11

$6470 \pm 120$

Lake marl with Anodonta.

St-1588. Mällingsmyr 12

$220 \pm 95$

Hard peat.

St-1590. Mällingsmyr 10

$7770 \pm 190$

Sand with peat layers.

St-1586. Mällingsmyr 13

5820 B.C.

Clay with stones and radicels.

$10,400 \pm 120$

8450 B.C.

\section{Sällmyr}

Samples under Ancylus shoreline at Sällmyr (57 $18^{\prime} \mathrm{N}$ Lat, $18^{\circ} 13^{\prime}$ E Long).

St-1556. Sällmyr 21

$8760 \pm 100$

Peat.

6810 B.C.

St-1553. Sällmyr 22

$8480 \pm 100$

Wood (log).

6530 B.C.

\section{Båticke}

Peat under Littorina shoreline at Båticke $\left(57^{\circ} 29^{\prime} \mathrm{N}\right.$ Lat, $18^{\circ} 14^{\prime} \mathrm{E}$ Long).

St-1624. Båticke 1

A.D. 1305

$645 \pm 120$

St-1722. Båticke B

$3930 \pm 105$

1980 B.C.

St-1591. Stigstäde

$4335 \pm 80$

2385 B.C.

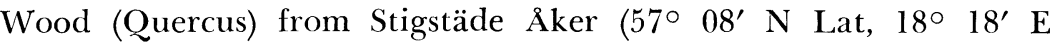


Long.). Locality was at first (in nineteenth century) supposed to be of interest for problems in connection with uplift of land.

St-1708. Kvarne A

$5780 \pm 85$

3830 B.C.

Clay under Littorina shoreline at Kvarne $\left(57^{\circ} 30^{\prime} \mathrm{N}\right.$ Lat, $18^{\circ} 38^{\prime} \mathrm{E}$ Long), Eskelhem. Coll. by C. G. Holdar, Gotland.

\section{Gurpe}

Sandy peat under Ancylus shoreline at Gurpe $\left(57^{\circ} 27^{\prime} \mathrm{N}\right.$ Lat, $18^{\circ} 38^{\prime}$ E Long).

\section{St-1560. Gurpe 8}

$7835 \pm 95$

St-1628. Gurpe 9

5885 в.c.

$7815 \pm 110$

5865 в.c.

\section{Snoder}

Peat and clay under Littorina shoreline at Snoder $\left(57^{\circ} 14^{\prime} \mathrm{N}\right.$ Lat, $18^{\circ} 13^{\prime}$ E Long).

\section{St-1585. Snoder 14}

$5240 \pm 85$

Peat covered with eolian sand.

St-1584. Snoder 15

$5995 \pm 85$

Lake marl under beach.

St-1583. Snoder 16 a

$7750 \pm 90$

Lower peat under beach.

5800 B.c.

St-1582. Snoder $16 \mathrm{~b}$

$6630 \pm 80$

Upper peat under beach.

4680 в.c.

\section{Helgmyr}

Peat under Littorina shoreline at Helgmyr $\left(57^{\circ} 33^{\prime} \mathrm{N}\right.$ Lat, $18^{\circ} 13^{\prime}$ E Long).

St-1619. Helgmyr 17

$6450 \pm 100$

Peat.

St-1620. Helgymr 18

$6615 \pm 100$

Peat, very compact.

4665 в.c.

St-1621. Helgmyr 19

$7380 \pm 90$

Peat and gyttja. 
St-1622. Helgmyr 20

$7005 \pm 140$

Peat and gyttja, very hard.

\section{Tomtmyr}

Peat and wood under Ancylus shoreline at Tomtmyr (57 $33^{\prime} \mathrm{N}$ Lat, $18^{\circ} 13^{\prime}$ E Long).

\section{St-1555. Tomtmyr 3}

$<250$

Log.

St-1557. Tomtmyr 4

$21,715 \pm 1300$

Peat. Age impossible to explain.

19,765 в.C.

St-1558. Tomtmyr 5

$<250$

Peat.

St-1641. Tomtmyr 30

$8905 \pm 100$

Peat.

6955 B.C.

St-1638. Tomtmyr 31

$9205 \pm 100$

Peat.

7255 B.c.

St-1634. Tomtmyr A

$9460 \pm 110$

Peat.

7510 B.C.

St-1635. Tomtmyr B

$8690 \pm 100$

Peat.

6740 B.C.

\section{Malms} Long).

Peat under Ancylus shoreline at Malms $\left(57^{\circ} 33^{\prime} \mathrm{N}\right.$ Lat, $18^{\circ} 31^{\prime} \mathrm{E}$

St-1564. Malms 6

$\mathbf{8 4 1 0} \pm \mathbf{1 1 5}$

Peat of Amblystegium.

6460 B.C.

St-1563. Malms 7

$\mathbf{8 4 1 0} \pm 145$

Peat.

6460 B.C.

\section{St-1611. Fröjel}

$9155 \pm 175$

7205 B.C.

Peat under Ancylus shoreline at Gåistes ( $57^{\circ} \mathrm{N}$ Lat, $18^{\circ} 15^{\prime}$ E Long). Made in order to compare with St-174 (Stockholm I), $9190 \pm 130$ B.P. 
Dynisse

Peat under Littorina shoreline at Dynisse $\left(57^{\circ} 16^{\prime} \mathrm{N}\right.$ Lat, $18^{\circ} 11^{\prime} \mathrm{E}$ Long), Sproge.

St-1609. Dynisse 20

$7025 \pm 180$

4 microscopical samples.

5075 B.C.

St-1610. Dynisse BP 13

$7400 \pm 155$

4 microscopical samples from another point.

5450 в.C.

\section{St-1612. Dynisse A}

$6415 \pm 80$

Peat.

4465 в.C.

St-1613. Dynisse B

$7260 \pm 100$

Peat.

5310 B.C.

\section{St-1616. Dynisse C}

$7815 \pm 90$

Peat.

St-1617. Dynisse D

$5895 \pm 80$

Peat.

3945 B.C.

St-1618. Dynisse E

$7125 \pm 95$

Peat.

\section{St-2089. Suoksjokk, Lappland}

$8120 \pm 90$

6170 B.C.

Stump found below $10 \mathrm{~m}$ of sediments at the river Luleälv $\left(66^{\circ} 3 \mathrm{I}^{\prime} \mathrm{N}\right.$ Lat, $20^{\circ} 31^{\prime}$ E Long), county of Norrbotten. Coll. by I. Hector, Swedish State Power Board; subm. by G. Lundqvist.

$7545 \pm 180$

\section{St-1916. Tärnasjön}

5595 B.C.

Sediment from Tärnasjön $\left(60^{\circ} 56^{\prime} \mathrm{N}\right.$ Lat, $15^{\circ} 29^{\prime} \mathrm{E}$ Long), at alt of $603 \mathrm{~m}$ in county of Västerbotten, 74 to $81 \mathrm{~cm}$ below surface of sediment. Coll. and subm. by Gunnar Hoppe, Dept. of Physical Geography, Univ. of Stockholm, Stockholm.

\section{B. Volcanic Ash}

Investigation made in order to date horizons with volcanic ash in peat-bogs from Sweden, Norway and the Faroes. Samples coll. and subm. by Christer Persson, Geol. Survey of Sweden, Stockholm. Samples indicated with "T" are only dried before preparation. 
Klockamyren $\left(62^{\circ} 18^{\prime}\right.$ N Lat, $12^{\circ} 28^{\prime}$ E Long $)$

St-2106. Klockamyren 71

St-2107. Klockamyren 23

St-2108. Mellstabromossen 47

(60 $30^{\prime} \mathrm{N}$ Lat, $15^{\circ} 22^{\prime} \mathrm{E}$ Long).

St-2119. Grövelsjömyren 25

(620 $05^{\prime} \mathrm{N}$ Lat, $12^{\circ} 23^{\prime} \mathrm{E}$ Long).

Sundhornsmossen $\left(60^{\circ} 33^{\prime}\right.$ N Lat, $15^{\circ} 38^{\prime}$ E Long $)$

St-1604. Sundbornsmossen 23

St-1605. Sundbornsmossen 182

NORWAY

Sjetnemyren ( $63^{\circ} 21^{\prime}$ N Lat, $11^{\circ} 03^{\prime}$ E Long)

St-1996. Sjetnemyren 25 T

St-2064. Sjetnemyren 25

St-1997. Sjetnemyren 59 T

St-2063. Sjetnemyren 59

Setranmyren ( $62^{\circ} 27^{\prime}$ N Lat, $\left.08^{\circ}{04^{\prime}}^{\text {E Long }}\right)$

St-1998. Setranmyren 45 T

St-1999. Setranmyren $177 \mathrm{~T}$

St-2065. Setranmyren 45

St-2066. Setranmyren 177 $\mathbf{3 7 0 0} \pm \mathbf{7 5}$

1750 B.C.

$575 \pm 65$

A.D. 1375

$1090 \pm 65$

A.D. 860

$1535 \pm 70$

A.D. 415
A.D. 1375

$575 \pm 70$

A.D. 1460

$490 \pm 65$

$1100 \pm 65$ A.D. 850

$1100 \pm 70$

A.D. 850

A.D. 1445

$505 \pm 65$

$2775 \pm 70$

825 B.C.

$330 \pm 80$

A.D. 1620

$\mathbf{3 0 5 0} \pm \mathbf{1 0 0}$

1100 B.C.

Kristiansundsmyren $\left(63^{\circ} \mathbf{0 4}^{\prime} \mathbf{N}\right.$ Lat, $07^{\circ} 50^{\prime}$ E Long $)$

St-2057. Kristiansundsmyren 34-36

$965 \pm 70$

A.D. 985 
St-2059. Kristiansundsmyren 68-70

St-2084. Kristiansundsmyren 91

St-2087. Kristiansundsmyren 158

THE FAROES

Saksunmyren $\left(62^{\circ} 12^{\prime} \mathrm{N}\right.$ Lat, $^{0} 7^{\circ} \mathbf{0 4}^{\prime}$ W Long $)$

St-2132. Saksunmyren 11

St-2133. Saksunmyren 55

St-2134. Saksunmyren 65

Myrarnar $\left(62^{\circ}{09^{\prime}}^{\prime}\right.$ 07 $^{\prime \prime}$ N Lat, $07^{\circ} 0^{\prime}$ W Long $)$

St-2135. Myrarnar 2:21

St-2136. Myrarnar 2:75

St-2139. Myrarnar 2:84

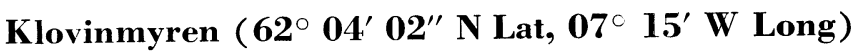

St-2140. Klovinmyren 37

St-2151. Klovinmyren 50

St-2155. Klovinmyren 94

St-2156. Klovinmyren 105

St-2078. Klovinmyren 280
$2730 \pm 70$

780 в.C.

$3600 \pm 80$

1650 B.c.

$6875 \pm 90$

4925 B.C.

$1585 \pm 70$

A.D. 365

$3075 \pm 100$

1125 B.c.

$3350 \pm 130$

1400 в.C.

A.D. 1050

$900 \pm 120$

$3520 \pm 105$

1570 B.C.

$3650 \pm 560$

1700 B.C.

\section{Shetland}

This series forms part of study of glacial and vegetational history of Shetland and of sealevel changes in area. Samples were collected mainly by Professor G. Hoppe, Dept. of Physical Geography, Univ. of Stockholm, Professor M. Fries, Royal College of Forestry, Stockholm, and Mr. A. Häggblom, Dept. of Physical Geography, Univ. of Stockholm, during an expedition in 1964. Subm. by Professor Hoppe. 


\section{Whalsay series}

Submarine peat and wood from Symbister, Whalsay $\left(60^{\circ} 20^{\prime} 05^{\prime \prime} \mathrm{N}\right.$ Lat, 01 02' W Long) (Hoppe, 1965).

\section{St-1552. Sy I}

$6030 \pm 80$

Peat from $8.9 \mathrm{~m}$ below present high-water level.

4080 B.c.

\section{St-1812. $13 / \mathbf{N} /$ Bottom}

$5945 \pm 230$

3995 в.c.

Peat from the lowest few $\mathrm{cm}$ of $20-\mathrm{cm}$ thick peat layer, depth below high-water level $9.0 \mathrm{~m}$.

\section{St-1811. 13/N/Top}

$5455 \pm 170$

Peat from top few cm 3505 B.c. depth below high-water level $8.8 \mathrm{~m}$.

\section{St-1809. Sy $/ \mathrm{W} / \mathbf{N 1 4}$}

$6970 \pm 100$

Wood (probably from Salix sp) embedded in peat lay, depth below high-water level $8.8 \mathrm{~m}$.

\section{St-1925. Sy/14/N}

$6670 \pm 100$

Peat from same place as St-1890, depth below high-water level $8.8 \mathrm{~m}$.

\section{Tresta series} Long).

Peat and wood in peat from land, Tresta $\left(60^{\circ} 15^{\prime} \mathrm{N} \mathrm{Lat,} 01^{\circ} 20^{\prime} \mathrm{W}\right.$

\section{St-1608. 264/Vb}

$5145 \pm 90$ layer.

3195 B.C.

\section{St-1607. Vd/1}

$5865 \pm 95$ layer in St-1608 and St-1714).

\section{St-1714. Tr/311}

$11,135 \pm 135$

9185 B.c.

Peat from 1-cm thick layer, below 290-cm thick layer of peat (same as St-1608) and $43 \mathrm{~cm}$ of mineral soil.

\section{St-1713. Loch of Wick}

$5470 \pm 100$

From $60^{\circ} 09^{\prime} \mathrm{N}$ Lat, $01^{\circ} 13^{\prime} \mathrm{W}$ Long. Lowermost $2 \mathrm{~cm}$ of $230-\mathrm{cm}$ thick layer of peat on a slope, just $S$ of Loch of Wick. 
Lake sediment series

Risk of contamination by old carbon cannot be excluded (for instance in St-1757) and this risk is now object of investigations.

St-1554. Sand Water

$10,055 \pm 300$

Site $\left(60^{\circ} 17^{\prime} \mathrm{N}\right.$ Lat, $01^{\circ} 20^{\prime} \mathrm{W}$ Long), at alt of $37 \mathrm{~m}$. From 125 to $128 \mathrm{~cm}$ below surface of sediment.

$9725 \pm 265$

St-1595. Stanevatstoe Loch

7775 B.c.

Site $\left(60^{\circ} 16^{\prime} \mathrm{N}\right.$ Lat, $01^{\circ} 20^{\prime} \mathrm{W}$ Long), at alt of ca. $80 \mathrm{~m}$. From 382 to $389 \mathrm{~cm}$ below surface of sediment.

Upper Loch of Brouster $\left(\begin{array}{llll}60^{\circ} & 15^{\prime} & \mathbf{N} \text { Lat, } 01^{\circ} 36^{\prime} \mathrm{W}\end{array}\right.$

Long), at alt of ca. $3 \mathrm{~m}$

St-1606. Br 400

$8760 \pm 250$

6810 B.C.

From 400 to $404 \mathrm{~cm}$ below the surface of the sediment.

St-1763. UB/14

$9670 \pm 540$

From 444 to $450 \mathrm{~cm}$ below surface of sediment.

7720 B.C.

$\begin{array}{ll} & 15,080 \pm 850 \\ \text { St-1757. Lower Loch of Brouster } & \mathbf{3 1 , 1 3 0} \text { B.C. }\end{array}$

From $60^{\circ} 15^{\prime} \mathrm{N}$ Lat, $01^{\circ} 36^{\prime} \mathrm{W}$ Long. Tidal lake from 345 to 350 $\mathrm{cm}$ below surface of sediment.

\section{St-1810. Strom Loch \\ $2615 \pm 140$ \\ 665 в.C.}

From $60^{\circ} 14^{\prime} \mathrm{N}$ Lat, $01^{\circ} 17^{\prime} \mathrm{W}$ Long. Tidal lake. From 300 to 302 $\mathrm{cm}$ below surface of sediment.

Loch of Clickimin $\left(60^{\circ} 09^{\prime} \mathrm{N}\right.$ Lat, $01^{\circ} 10^{\prime} \mathrm{W}$ Long $)$

$\begin{array}{ll}\text { St-1639. Loch of Clickimin } 140 & 9620 \pm 750 \\ & \mathbf{7 6 7 0} \text { B.c. }\end{array}$

At sealevel, but normally not a tidal lake. From 137 to $140 \mathrm{~cm}$ below surface of sediment.

\section{St-1640. Loch of Clickimin 180}

From 177 to $180 \mathrm{~cm}$ below surface of sediment.

\section{St-1559. Loch of Brow}

Located (59 $56^{\prime} \mathrm{N}$ Lat, $01^{\circ} 19^{\prime} \mathrm{W}$ Long) at alt of ca. $1 \mathrm{~m}$. From 198 to $201 \mathrm{~cm}$ below surface of sediment.
$12,090 \pm 900$

10,140 в.C.

$9945 \pm 250$

7995 B.c. 


\section{Other Countries}

\section{Azores series}

AzORES

Material from the Azores, Portugal. Coll. and subm. by Magnus Fries, Royal College of Forestry, Stockholm.

\section{St-2068. Azores 1}

$$
3435 \pm 75
$$

\section{St-2077. Azores 2}

Two pieces of fossil wood, from Juniperus brevifolia from Sao

$1815 \pm 65$ Miguel (32 $52^{\prime} \mathrm{N}$ Lat, $25^{\circ} 4 \overline{7}^{\prime} \mathrm{W}$ Long).

\section{St-2109. Azores 3}

$845 \pm 200$ W Long).

Sediment core from Lagon Azul, Sao Miguel (37॰ $52^{\prime} \mathrm{N}$ Lat, $25^{\circ} 47^{\prime}$

\section{St-2114. Azores 4}

Sediment core from Lagoa das Furnas, Sao Miguel $\left(37^{\circ} 45^{\prime} \mathrm{N}\right.$ Lat, $25^{\circ} 20^{\prime}$ W Long).

\section{St-2117. Azores 5}

Sediment core from Lagona Raza, Sao Miguel $\left(37^{\circ} 50^{\prime} \mathrm{N}\right.$ Lat, $25^{\circ}$ $46^{\prime}$ W Long).

\section{St-2118. Azores 6}

Sediment core from Lagoa do Capita, Ilha do Pico $\left(38^{\circ} 28^{\prime} 05^{\prime \prime} \mathrm{N}\right.$ Lat, $28^{\circ} 19^{\prime} \mathrm{W}$ Long).

\section{St-1800. Hvammkot 20}

\section{ICELAND}

Peat from peat bog of Hvammkot $\left(66^{\circ} \mathrm{N}\right.$ Lat, $20^{\circ} \mathrm{W}$ Long), on Iceland. Dating of peat growth $200 \mathrm{~cm}$ below surface. Sample coll. and subm. by Gudfinna Ragnarslottir, Dept. of Quaternary Geology, Univ. of Stockholm, Stockholm.

\section{Sigluvik series}

Dating of volcanic ash layer. Peat from peat bog of Sigluvik $\left(65^{\circ}\right.$ $45^{\prime} \mathrm{N}$ Lat, $18^{\circ} 00^{\prime} \mathrm{W}$ Long), Iceland. Coll. and subm. by Gudfinna Ragnarsdottir.

St-1799. Sigluvik 60

$525 \pm 65$

$60 \mathrm{~cm}$ below surface. 
St-2137. Sigluvik 2

4115 B.C.

$210 \mathrm{~cm}$ below surface.

\section{Keflavik series}

Peat from peat bog of Keflavik (65 $45^{\circ} \mathrm{N}$ Lat, $19 \circ 30^{\prime} \mathrm{W}$ Long), Hegranes, Iceland. Coll. and subm. by Gudfinna Ragnarsdottir.

St-2138. Keflavik 2.7-3.0

$5535 \pm 120$

3585 B.C.

270 to $300 \mathrm{~cm}$ below surface. Dating Sphagnum peat period.

$\mathbf{5 6 5 0} \pm \mathbf{8 5}$

St-1797. Keflavik 2.6-3.9

3700 B.C.

360 to $390 \mathrm{~cm}$ below surface. Dating of Betula rise.

$7280 \pm 150$

St-1798. Keflavik 5.1-5.3

5330 B.C.

510 to $530 \mathrm{~cm}$ below surface. Bottom layer.

II. ARCHAEOLOGIC SAMPLES

A. Sweden

\section{Krankmårtenhögen series}

Charcoal and resin caulkings from gravefield at lake Storsjön $\left(62^{\circ}\right.$ $46^{\prime} 40^{\prime \prime} \mathrm{N}$ Lat, $13^{\circ} 13^{\prime} 23^{\prime \prime} \mathrm{E}$ Long), Storsjö parish, Härjedalen. One grave contains metal artifacts which cannot be dated archaeologically. Resin was used as tightening material in wooden boxes filled with cremated bones. There were also a lot of stone artifacts in some graves. See Krankmårtenhögen series (Stockholm VI). Samples coll. by Göran Stolpe; subm. by B. Ambrosiani, Royal Office of Antiquities.

\section{St-1834. V :A2}

$6325 \pm 90$

Charcoal (with cremated bones).

St-1835. VI:A52

$1555 \pm 100$

Charcoal under burnt stones of hearth. A.D. 395
St-1836. VII:A10
$2130 \pm 90$
180 B.C.
St-1837. VIII:A33
Charcoal (with cremated bones).
$\mathbf{3 9 3 0} \pm \mathbf{8 0}$
1980 B.C.

Charcoal from cooking pit under pieces of brittle burnt stones. 
St-1841. $\quad X: A 59$

Charcoal (with cremated bones).

St-1842. XI:A1

$1855 \pm 160$

St-1843. XII :A3

A.D. 95

St-1844. XIII:A4

$2245 \pm 100$

295 B.C.

$2330 \pm 70$

380 в.с.

St-1845. XIV :A37

Resin (with cremated bones).

$2110 \pm 70$

160 B.C.

\section{Kårtjejaure series}

Charcoal from some of the hearths of the site Kårtjejaure 1371 (62 $29^{\prime} \mathrm{N}$ Lat, $18^{\circ} 17^{\prime} \mathrm{E}$ Long), Stora Lule river, Gällivare parish, Lappland. Only one culture layer was found at site containing stone artifacts, nails, broken pieces, ceramics, glass, etc. Coll. by Kerstin Bergengren and subm. by Björn Ambrosiani.

\author{
St-1566. 1371:413 \\ St-1567. $1371: 419$ \\ St-1568. 1371:Z 6 \\ St-1569. 1371:V 16 \\ St-1570. 1371:AF 16 \\ St-1571. 1371:AB 12 \\ St-1572. 1371:AA 14 \\ St-1573. 1371:AA 12 \\ St-1575. 1371:X 8 \\ St-1576. 1371:Z 10
}

\section{St-1838. Jörlanda IX}

$$
740 \pm 65
$$
A.D. 1210

$$
<250
$$

$3650 \pm 70$

1700 B.c.

A.D. 1520

$$
430 \pm 65
$$

A.D. 1670

$280 \pm 65$

$6330 \pm 80$

4380 в.c.

A.D. 1580

$370 \pm 65$

$1925 \pm 70$

A.D. 25

$1995 \pm 70$

45 B.C.

$920 \pm 85$

A.D. 1030

$4500 \pm 170$

2550 B.c.

Charcoal found in passage to a dolmen, in yellow sand under stone accumulation, Jörlanda parish (58 ${ }^{\circ} \mathrm{N}$ Lat, $11^{\circ} 45^{\prime} \mathrm{E}$ Long), Bohuslän. Coll. by Ingegerd Särlvik; subm. by B. Ambrosiani. 


\section{Luotosuollo series}

Charcoal from site on Luotosuollo, islet in eastern part of lake Satisjaure (62 $27^{\prime} \mathrm{N}$ Lat, $18^{\circ} 55^{\prime} \mathrm{E}$ Long), Gällivare parish, Norrbotten. Samples coll. by K. Bergengren; subm. by Björn Ambrosiani. Archaeological finds from site are partly of Stone Age type, partly remains of Lapp settlements of present time.

St-1851. L 1

Hearth 10 .

St-1852. L 2

Charcoal from circle of stones of unknown use.

St-1853. L 3

$<250$

Hearth 6 .

St-1854. L 4

$<\mathbf{2 5 0}$

Hearth 1

St-1855. L 5

$\mathbf{3 9 2 0} \pm \mathbf{1 2 0}$

Hearth 9, coal of Pinus silvestris.

St-1856. L 6

Coal from Pinus silvestris in layer of brittle burnt stones.

$1760 \pm 110$

St-1857. L 7

A.D. 190

Hearth 8, coal from Betula sp.

St-1858. L 8

Hearth 7, coal from Betula sp.

St-1859. L 9 a

Charcoal from Betula immediately below the peat in pit No. 1 .

$305 \pm 70$

St-1860. L 9 b

A.D. 1645

Bottom layer in pit No. 1.

$3145 \pm 100$

1195 B.c.

St-1867. Trälösa 1

Charcoal from mound of brittle burnt stones at settlement at Trälösa (59 55' N Lat, $17^{\circ} 43^{\prime}$ E Long), Vaksala parish, Uppland. Coll. by Sten Rentzhog; subm. by Björn Ambrosiani. No datable finds were made. Adjacent to mound are remains of what has probably been small house. 
Ändesta series

Charcoal from Ändesta $\left(59^{\circ} 35^{\prime} \mathrm{N}\right.$ Lat, $16^{\circ} 45^{\prime} \mathrm{E}$ Long), Kungsåra parish, Västmanland. Samples coll. by Sten Rentzhog; subm. by U. E. Hagberg, Royal Office of Antiquities.

\title{
St-1868. 287515
}

$1335 \pm 70$

From cairn of large round stones in middle of gravefield, mostly of brittle burnt stones. In the cairn, ceramics (of general rough type) and burnt bones were found.

St-1869. 312527

$2695 \pm 100$

745 B.c.

From mound of brittle burnt stones, gravel or larger round stones. A find of a small bronze saw was made.

\section{Bågede series}

Charcoal from grave at Bågede (64 $21^{\prime} \mathrm{N}$ Lat, $14^{\circ} 49^{\prime} \mathrm{E}$ Long), Frostviken parish, Jämtland. Round barrow with layer of stones. Coll. by Elisabeth Allard; subm. by Björn Ambrosiani.

\section{St-1599. Bågede 1}

$1130 \pm 60$

Layer of charcoal on level of stone layer.

\author{
A.D. 820
}

St-1601. Bågede 2

$$
1045 \pm 65
$$

St-1596. Bågede 3

A.D. 905

$1100 \pm 70$

Layer of charcoal on original ground surface under stones.

\section{Bölensvattnet series}

Charcoal from site at Lake Bölensvattnet $\left(63^{\circ} 59^{\prime} \mathrm{N}\right.$ Lat, $16^{\circ} 26^{\prime} \mathrm{E}$ Long), Bodum parish, Ångermanland. Finds consist of stone artifacts and small pieces of ceramics from only one cultural layer. Samples coll. by Elisabeth Allard; subm. by Björn Ambrosiani.

St-1656. 1356:1

St-1667. 1356:2

Hearth, charcoal layer above stone layer.

St-1668. 1356:3

St-1657. 1356:4

Hearth, thick layer of charcoal under stones.

St-1661. 1356:5

Small pit with charcoal, brittle burnt stones and burnt bones.
$1035 \pm 75$

A.D. 915

$$
<\mathbf{2 5 0}
$$

A.D. 750

$$
1075 \pm 75
$$

A.D. 875

$4920 \pm 80$ 2970 B.C. 
St-1663. 1356:6

55 B.c.

Layer of charcoal in upper level of cultural layer.

St-1669. 1356:7

$1035 \pm 80$

A.D. 915

$1185 \pm 80$

St-1664. 1356:8

A.D. 765

Hearth $1356 \mathrm{~B}$, thick layer of charcoal under stones.

\section{Lesjön series}

Charcoal from sites S-101 Grananäset and S-104 Lemnäset at Lake Lesjön (63 $38^{\prime} \mathrm{N}$ Lat, $17^{\circ} 03^{\prime} \mathrm{E}$ Long), Bodum parish, Ångermanland. Finds of stone artifacts, ceramics and pieces of some bone artifacts. Coll. by Elisabeth Allard; subm. by Björn Ambrosiani.

St-1602. S101:1

$2245 \pm 70$

St-1603. S101:2

295 B.C.

Thin layer of charcoal on top of culture layer.

St-1614. S101:3

$1615 \pm 65$ A.D. 335

\section{St-1598. S101:4}

Thin layer of charcoal on bottom of culture layer.

St-1647. S104:1

$$
\begin{aligned}
& 3400 \pm 110 \\
& 1450 \text { B.C. } \\
& 4155 \pm 145 \\
& 2205 \text { B.C. }
\end{aligned}
$$

Hearth 1, among and under stones.

St-1615. A104:2

$2245 \pm 85$ 295 B.C.

$$
2320 \pm 65
$$

370 в.c.

Hearth 2, under stones.

St-1648. S104:3

$5765 \pm 110$

3815 B.c.

Under concentration of burnt stones.

St-1649. S104:4

$3735 \pm 100$ 1785 B.C.

$5285 \pm 130$

St-1650. S104:5

St-1651. S104:6

$6345 \pm 180$ 4395 в.c.

Thin layer of charcoal partially covered by sand under thick cultural layer. 
St-1652. S104:7

$$
2830 \pm 70
$$

St-1653. A104:8

Thin layer of charcoal in cultural layer.

St-1654. S104:9

Charcoal among and under pieces of brittle burnt stones.

St-1655. S104:10

$5440 \pm 115$

Small pit with charcoal and burnt bones.

3490 B.C.

\section{Älvenäs series}

Charcoal found in bottom of cairn in a gravefield at Nor River

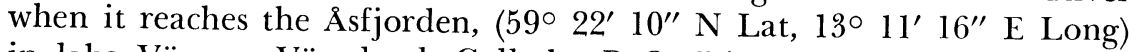
in lake Vänern, Värmland. Coll. by P.-O. Ringquist; subm. by Björn Ambrosiani.

St-1731. A8:IV

St-1732. A7:II

St-1733. A7 :III

St-1734. A9:I

St-1736. A11:V

St-1737. A11:VI
A.D. 1120

$$
\mathbf{8 3 0} \pm \mathbf{8 0}
$$

$$
<250
$$

A.D. 1580

$$
370 \pm 70
$$

A.D. 1620

$$
330 \pm 65
$$

$<\mathbf{2 5 0}$

$<250$

$2615 \pm 75$

665 B.c.

\section{St-1735. Hammar $1^{3}$}

Charcoal found together with cremated bones and pottery under one of the stones in a stone circle. Solberga parish $\left(57^{\circ} 53^{\prime} \mathrm{N} \mathrm{Lat,} 11^{\circ} 50^{\prime} \mathrm{E}\right.$ Long), Bohuslän. Coll. by Hille Jaanusson; subm. by Björn Ambrosiani.

\section{Igelsta series I}

Charcoal from plain, cairn-like, brittle-burnt heap of stones at Igelsta (59 $10^{\prime} \mathrm{N}$ Lat, $17^{\circ} 40^{\prime} \mathrm{E}$ Long), Östertälje parish, Södermanland. Grave, $10 \mathrm{~m}$ in diam, covered crevice of $1 \mathrm{~m}$ depth. Charcoal and burned bones were found under stones. Other finds were a razor, knife, tweezers and a button, all in bronze. Coll. by Åke Hyenstrand; subm. by Björn Ambrosiani.

St-1738. $\quad 707: 335,5-51$

$305 \pm 75$

A.D. 1645 


\begin{tabular}{|c|c|c|}
\hline & & $\begin{array}{r}880 \\
\text { A.D. } 1070\end{array}$ \\
\hline St-1739. & $705: 335-60$ & $\begin{array}{r}\text { A.D. } 1060 \\
490 \pm 70\end{array}$ \\
\hline$t-1741$. & $704: 336-70$ & A.D. 1460 \\
\hline
\end{tabular}

\section{Igelsta series II}

In connection with excavation of group of Bronze Age burial cairns on Igelsta mountain in östertälje parish, a dwelling-place was found and excavated on a low terrace immediately $S$ of mountain foot. A floorlike stratum of clay and stones was discovered. Stratum was surrounded by four small pits in sand, to some extent lined with stones and filled with soot and charcoal. They were possibly remains of firesides or cooking pits. Charcoal was taken for radiocarbon dating. On the dwelling place, pottery and burned bones were found. The most numerous finds, however, consisted of burned pieces of clay, probably once packed on plaited walls of sticks. The find of a hut in a probable Bronze Age milieu is almost unique in central Sweden and a dating is extraordinarily valuable.

$\begin{array}{llr}\text { St-1846. } & 690: 227,60-61 & <250 \\ & & 2365 \pm 70 \\ \text { St-1848. } & 640: 366,-150 & 415 \text { B.c. } \\ & & 2775 \pm 70 \\ \text { St-1849. } & 639: 362,-165 & 825 \text { B.c. } \\ & & 2570 \pm 70 \\ \text { St-1850. } & 642: 365,-125 & 620 \text { B.c. }\end{array}$

\section{Skagersvik series}

Charcoal from gravefield in Skagersvik $\left(58^{\circ} 59^{\prime} \mathrm{N}\right.$ Lat, $14^{\circ} 06^{\prime} \mathrm{E}$ Long), Amnehärads parish, Västergötland. Coll. by Inga Lundström; subm. by Björn Ambrosiani. Found with burned bones in cremation pit $\mathrm{Al}: \mathrm{F} 1$, in cremation layer A3:F5, A4:F9 and in urns A2:F2. Archaeological finds indicate Migration period and early Vendel Age. Under the finds was dark layer rich in flints that seem to belong to Mesolithic period.

\author{
St-1715. A4:F9 \\ St-1716. A3:F6 \\ St-1717. A3:F5 \\ St-1718. A2:F2 \\ St-1719. A1:F1
}

$1165 \pm 85$

A.D. 785

$$
<\mathbf{2 5 0}
$$

$1685 \pm 230$

A.D. 265

$1230 \pm 115$

A.D. 720

$1695 \pm 130$

A.D. 255 
Lossnen series

Continued from Stockholm IV. Charcoal, wood and resin from gravefield at Smalnäset, Lake Lossnen $\left(62^{\circ} 25^{\prime} \mathrm{N}\right.$ Lat, $12^{\circ} 51^{\prime} \mathrm{E}$ Long), Tännäs parish, Härjedalen. Coll. and subm. by H. Hvarfner, Royal Office of Antiquities, Stockholm.

\section{St-1720. Lossnen 6 \\ St-1723. Lossnen 12 \\ St-1724. Lossnen 16 \\ St-1725. Lossnen 23 \\ St-1726. Lossnen 25 \\ St-1727. Lossnen 31}

$$
\begin{gathered}
2100 \pm 65 \\
150 \text { B.C. }
\end{gathered}
$$

$1965 \pm 110$

15 B.c.

$6650 \pm 90$

4700 в.c.

$1875 \pm 90$

A.D. 75

$2430 \pm 70$

480 в.C.

$4485 \pm 150$

2535 B.c.

$5440 \pm 85$

3490 B.C.

St-1728. Tjärnbäcken

Charcoal found with burnt bones of Alces alces and Ursus arctos in a grave at Lake Lossnen (62 $25^{\prime} \mathrm{N}$ Lat, $12^{\circ} 51^{\prime} \mathrm{E}$ Long), Tännäs parish, Härjedalen. Subm. by H. Hvarfner.

\title{
Tjikkiträsk series
}

Charcoal from site at St. Tjikkiträsk ( $64^{\circ} 59^{\prime} \mathrm{N}$ Lat, $17^{\circ} 42^{\prime} \mathrm{E}$ Long), Stensele parish, Västerbotten. Subm. by H. Hvarfner.

\author{
St-1750. T 1 \\ St-1751. T 2 \\ St-1752. T 3 a \\ St-1753. $\quad$ T 7 a \\ St-1754. T 8 \\ St-1755. T 9
}


Rappasundet series

Charcoal from site at Hornavan $\left(66^{\circ} 04^{\prime} \mathrm{N}\right.$ Lat, $17^{\circ} 54^{\prime} \mathrm{E}$ Long), Arjeplog parish, Norrbotten. Subm. by Birgitta Hallgren and Harald Hvarfner.

St-626. Rappasundet 5

St-627. Rappasundet 6

St-632. Rappasundet 7

St-637. Rappasundet 8

St-1729. Rappasundet 9

St-641. Rappasundet 11

St-1730. Rappasundet 12

St-1742. Rappasundet 14

St-1743. Rappasundet 15

St-1744. Rappasundet 16

St-1745. Rappasundet 18

St-1746. Rappasundet 19

St-1747. Rappasundet 20
$2180 \pm 75$

230 B.C.

A.D. 1410

$540 \pm 65$

$2165 \pm 100$

215 B.C.

$1950 \pm 80$

O B.C.

$2235 \pm 100$

285 B.c.

$2235 \pm 80$

285 B.c.

$2080 \pm 90$

130 B.C.

$2495 \pm 70$

545 в.c.

$2290 \pm 75$

340 B.C.

$1705 \pm 75$

A.D. 245

$1955 \pm 75$

5 B.c.

$1995 \pm 75$

45 в.c.

$2695 \pm 70$

745 B.C.

\section{Vä series}

Charcoal from site at Vä $\left(56^{\circ} \mathrm{N}\right.$ Lat, $14^{\circ} \mathrm{E}$ Long), Skáne. Site has been occupying almost same area as later medieval town. About 30 small huts, pit huts or hut hollows, have been examined. Samples are collected from hearths of lowermost levels of pits. Coll. and subm. by Egon Thun, Royal Office of Antiquities, Stockholm.

\section{St-1783. 2696}

$970=65$

Trench XVIII, 2 to $6 \mathrm{~m}$, Hut 22, level $-180 \mathrm{~cm}$ (below present day surface), near bottom. 
St-1784. 2697

Trench XVIII, hut 22, level $-200 \mathrm{~cm}$.

St-1785. 2700

St-1785. 2700 $-150 \mathrm{~cm}$.

Trench 111, 9 to $11 \mathrm{~cm}$, Hut 27, just below hearthstones, level

St-1786. 2702

A.D. 750

$1200 \pm 70$

Trench XVII, 1 to $4 \mathrm{~m}$, Hut 19 from hearth at level of $154 \mathrm{~cm}$.

St-1787. 2707

$1165 \pm 70$

A.D. 785

Trench XIX, $29 \mathrm{~m}$, Hearth N, Hut 21, in lowermost culture layer of trench.

St-1788. 2708

A.D. 560

Trench XIX, 19 to $22 \mathrm{~m}$, Hut 21, from hearth at $+26.20 \mathrm{~m}$, ca. 10 $\mathrm{cm}$ over bottom of pit.

\section{St-1789. 2711}

$1035 \pm 65$

Trench XIX, 27.5 to $30.5 \mathrm{~m}$, Hut 21, from hearth at bottom.

\section{St-1790. 2713}

$1250 \pm 110$

St-1791. 2716

Trench V, $17 \mathrm{~m}$, Hut 8, from hearth at $-140 \mathrm{~cm}$.

St-1792. 2717

Trench V, Hut 8, from bottom layer of pit.

St-1793. 2718

Trench XVIII, 1 to $4 \mathrm{~m}$, Hut 19, from hearth at $-170 \mathrm{~cm}$.

$$
1345 \pm 65
$$

A.D. 605

St-1794. 2719

Trench XX, 2 to $8 \mathrm{~cm}$, lowermost cultural layer.

$1615 \pm 115$ A.D. 335

\section{Hammarby series}

Charcoal from hearths and postholes at Skälby $\left(59^{\circ} 41^{\prime} \mathrm{N}\right.$ Lat, $17^{\circ}$ 02 ' E Long), Hammarby parish, Uppland. Subm. by G. Arwidsson, Dept. of Nordic and Comparative Archeology, Univ. of Stockholm, and U. E. Hagberg, Royal Office of Antiquities, Stockholm. 
$1675 \pm 75$

St-2152. Hammarby 1

St-2153. Hammarby 2

St-2154. Hammarby 3
A.D. 275

$1845 \pm 70$

A.D. 105

$1855 \pm 90$

A.D. 95

\section{Örkelljunga series}

Charcoal from primitive iron melting place at östra Ringarp, $\left(56^{\circ}\right.$ $15^{\prime} \mathrm{N}$ Lat, $13^{\circ} 20^{\prime} \mathrm{E}$ Long), Skåne. Coll. by Sven Nöjd, Örkelljunga; subm. by H. Arbman, Historiska Museet, Univ. of Lund, Lund.

\section{St-1696. Örkelljunga 1}

St-1697. Örkelljunga 2
$650 \pm 65$

\section{A.D. 1300}

$740 \pm 65$

A.D. 1210

\section{Kårarp series}

Charcoal from hearth pits under cairn at Kårarp $\left(56^{\circ} 42^{\prime} \mathrm{N}\right.$ Lat, $12^{\circ} 52^{\prime}$ E Long), Övraby parish, Halland. Subm. by Lennart Lundborg, Hallands Mus., Halmstad.

St-1523. p 130123

St-1546. p 133120

St-1547. p 129135

St-1548. p 130136
$1995 \pm 100$

45 B.C.

$2015 \pm 70$

65 B.c.

$2050 \pm 65$

100 B.C.

$2395 \pm 100$

445 в.c.

\section{Eketorp series I}

Charcoal from Eketorp ring-fort $\left(56^{\circ} 02^{\prime} \mathrm{N}\right.$ Lat, $16^{\circ} 03^{\prime} \mathrm{E}$ Long), Gräsgårds parish, öland. Coll. within prehistoric fortress of Eketorp village. Fort and building foundations are built from limestone, which is local bedrock. Subm. by leader of excavation, Mårten Stenberger, Royal Office of Antiquities, Stockholm.

\section{St-1756. Eketorp I:1}

$1370 \pm 75$

Hearth on clay floor of lowest of three habitation levels. Finds include pottery, iron-bars, loom-weights and beads.

\section{St-1970. Eketorp A:1}

$1645 \pm 65$

Taken from ruined building A, fortress at Eketorp village. Finds include decorated pottery and bronze pin. 
St-1973. Eketorp II: 1

$$
1475 \pm 70
$$

bronze arrow head and glass fragments.

\section{St-1974. Eketorp III: 1}

$1545 \pm 65$

Taken from ruined building III. Finds include clasp, bronze and glass fragments.

St-1981. Eketorp IV:1

A.D. 490

$1460 \pm 65$

Taken from ruined building IV. Finds consisted of bronze rings.

\section{Eketorp series II}

Bone from same location as Eketorp series I. Coll. and subm. by Hans Sellstedt, Royal Office of Antiquities, Stockholm.

\section{St-2112. N 17:II}

Tibia of Homo.

St-2113. M 17:III

Ribs of Homo.

\section{St-2115. S 13:II}

Vertebrae and ribs of Homo.

\section{St-1961. Västerås 1}

Bone of Phoca vitulina in site from Stone Age near Västerås $(590$ $44^{\prime} \mathrm{N}$ Lat, $16^{\circ} 30^{\prime} \mathrm{E}$ Long), Västmanland.

\section{St-1976. Agnsjön}

$3660 \pm 115$

E Long site at Agnsjön (63 $33^{\prime} \mathrm{N}$ Lat, $17^{\circ} 54^{\prime}$ $\mathrm{E}$ Long). Anundsjö parish, Ångermanland. Investigation made in order to get opportunity to date Stone Age settlement. Subm. by Hans Sellstedt.

\section{Skede mosse series}

Bone from drained fen, Skede mosse $\left(56^{\circ} 05^{\prime} \mathrm{N}\right.$ Lat, $16^{\circ} 45^{\prime} \mathrm{E}$ Long), Gärdslösa parish, öland. See Skede mosse series in Stockholm VI. Coll. and subm. by Hans Sellstedt.

St-1924. 1003 A

St-1930. 1003 B

$$
1760 \pm 70
$$

A.D. 190

$$
1760 \pm 70
$$

A.D. 190

Femur of calf. St-1924 is upper part of bone and St-1930 is lower part of same bone. 
St-1975. 307

Humerus of horse.

St-1977. 1018

Humerus of pig.

St-1971. 805

Femur of Homo.

St-1978. 118

Ribs of horse.
$1935 \pm 75$

A.D. 15

$1955 \pm 70$

5 B.C.

$1420 \pm 65$

A.D. 530

$<\mathbf{2 5 0}$

\section{Sörby Tall series}

Charcoal from Iron Age settlement at Sörby Tall $\left(56^{\circ} 50^{\prime} \mathrm{N}\right.$ Lat, $16^{\circ} 45^{\prime}$ E Long), Gärdslösa parish, Öland. Subm. by U. E. Hagberg and Margareta Beskow, Royal Office of Antiquities, Stockholm.

St-1543. House II:A $33: F \quad 144$

From a hearth.

St-1544. House II:A 34:F 136

From a post hole.

St-1780. House II:F 133

$1695 \pm 75$

A.D. 255

$$
1655 \pm 70
$$

A.D. 295

$$
1400 \pm 70
$$

A.D. 550

From a clay level, which might have been a floor in the house.

St-1781. House II:A 10:F 155

$1370 \pm 85$

A.D. 580

From a post hole in same settlement as St-1543 and St-1544.

St-1782. House II:A 25:F 128

$1325 \pm 65$

A.D. 625

From a hearth in same settlement as St-1543 and St-1544.

St-1779. House I:A 13

$<\mathbf{2 5 0}$

From a supposed garbage-pit.

St-1866. F 201

$1835 \pm 75$

A.D. 115

\section{Ormöga series I}

Charcoal from Iron Age settlement in Bredsätra $\left(56^{\circ} 52^{\prime} \mathrm{N}\right.$ Lat, $16^{\circ} 48^{\prime}$ E Long), öland. subm. by U. E. Hagberg.

St-1540. F 21

$1565 \pm 70$

Probably the roof from a house. 
St-1862. A 5

$$
1665 \pm 70
$$

A.D. 285

St-1863. Hearth 6

$1725 \pm 70$

St-1864. A 21

A.D. 225

$1595 \pm 90$

St-1865. A 67

A.D. 355

$1460 \pm 65$

Ormöga series II

Bone from same site as Ormöga Series I. Samples coll. and subm. by Hans Sellstedt.

\section{St-2046. $\quad 0118$}

$1230 \pm 80$

St-2048. O 92

D. 720

$2080 \pm 105$

Petrosum of Greenland seal. Comment (H.S.): dating has shown that Greenland seal survived in the Baltic until at least 2000 B.P.

\section{Västerhus series}

Human bones, mainly femora and ribs, from 12 individuals in early Medieval cemetery at Westerhus chapel (63 $12^{\prime} \mathrm{N}$ Lat, $14^{\circ} 28^{\prime} \mathrm{E}$ Long), Frösö parish, Jämtland. Coll. by N. Lagerholm; subm. by N.-G. Gejvall, Royal Office of Antiquities, Stockholm. Anthropology and building phases discussed by Gejvall (1960). Present dating in order to check results in this thesis and in odontological thesis by Svärdstedt (1966). Comment (N.-G. G.): from historical and architectural point of view vital phase of this cemetery has developed in $\mathrm{E}$ to $\mathrm{W}$ direction from A.D. 1070 to A.D. 1350 , which is in very good agreement with $\mathrm{C}^{14}$ dates.

St-2141. F 54

St-2142. F 225

St-2143. F 104

St-2144. F 171

St-2145. F 181

St-2146. F 117

St-2147. F 9
$740 \pm 65$

A.D. 1240

A.D. 1300

$$
650 \pm 65
$$

$645 \pm 85$

A.D. 1305

$$
1010 \pm 65
$$

A.D. 940

$$
680 \pm 60
$$

A.D. 1270

$$
740 \pm 60
$$

A.D. 1210

A.D. 1185 
St-2148. F 177

St-2149. F 32

St-1909. $\quad 555$ A

St-1923. $\quad 555$ B

St-1919. 91

$750 \pm 65$

A.D. 1200

$830 \pm 60$

A.D. 1120

$880 \pm 65$

A.D. 1070

$775 \pm 70$

A.D. 1175

A.D. 1075

$875 \pm 65$

$465 \pm 65$

A.D. 1485

St-1660. Örlen

Wood from dug-out canoe found in lake örlen, Fagersanna $\left(58^{\circ}\right.$ $28^{\prime} \mathrm{N}$ Lat, $14^{\circ} 18^{\prime} \mathrm{E}$ Long), Karlsborg, Västergötland. Subm. by Bo Gräslund, Royal Office of Antiquities, Stockholm and A. Pohl, Karlsborgs Mus. Karlsborg.

St-1565. Västerljung $\mathbf{F} 134$

$2430 \pm 140$

480 в.C.

Scrapings from ceramics (pottery) found in Tuna $\left(58^{\circ} 54^{\prime} \mathrm{N} \mathrm{Lat}, 17^{\circ}\right.$ $28^{\prime}$ E Long), Västerljung parish, Södermanland. See Västerljung series (Stockholm VI). Subm. by Bo Gräslund.

\section{St-1861. Selånger F15:12}

$1545 \pm 65$

Charcoal from Grave No. 12 in Gravefield No. 43 at Granlo $\left(62^{\circ} 15^{\prime}\right.$ $\mathrm{N}$ Lat, $17^{\circ} 16^{\prime} \mathrm{E}$ Long), in Selånger parish, Medelpad. Subm. by M. Biörnstad, Royal Office of Antiquities.

St-1808. Site 792 (the pot of Laisan)

$3025 \pm 80$

1075 B.C.

Crushed sherds $(60 \mathrm{~g})$ from asbestos ceramics found at Lake Varris, (64 $42^{\prime} \mathrm{N}$ Lat, $16^{\circ} 24^{\prime} \mathrm{E}$ Long), Vilhelmina parish, Lapplund. See Malgomaj-Varris Series (Stockholm VI). Subm. by Astrid Linder, Royal Office of Antiquities.

\section{Havor series}

Charcoal from Lilla Havor (57 $17^{\circ} 13^{\prime} \mathrm{N}$ Lat, $18^{\circ} 19^{\prime} \mathrm{E}$ Long), Hablingsbo parish, Gotland. Subm. by G. Arwidsson, Dept. of Nordic and Comparative Archeology, Univ. of Stockholm, Stockholm.

St-1578. LH 1

$1995 \pm 65$

45 в.c.

St-1580. LH 2

$1945 \pm 70$

St-1592. LH 3

A.D. 5

$2050 \pm 100$

100 B.C. 


\section{St-1593. LH 4}

$$
2035 \pm 70
$$

85 в.c.

\section{St-1676. Mästermyr C 9903}

$1020 \pm 80$

Bees wax found in the bog Mästermyr $\left(57^{\circ} 10^{\prime} \mathrm{N}\right.$ Lat, $18^{\circ} 13^{\prime} \mathrm{E}$ Long), Hemse parish, Gotland. Subm. by G. Arwidsson.

\section{St-1712. Stads-Gånsta 4}

Charcoal from brittle-burnt cairn at Stads-Gånsta $\left(59^{\circ} 35^{\prime} \mathrm{N}\right.$ Lat, $17^{\circ} 15^{\prime}$ E Long), Vårfrukyrka parish, Uppland. Coll. by Louise Cederschiöld; subm. by Mats Malmer, Royal Office of Antiquities, Stockholm.

\section{Gotland series II}

Samples from island of Gotland in the Baltic. Coll. and subm. by G. Lundqvist, Geol. Survey of Sweden, Stockholm (Lundqvist, 1965). See Gotland series I, Geologic Samples, Sweden. There are two bog castles (Långmyrborgen and Vasstädemyrborgen), the big lake dwelling-place Bulverket in lake Tingstäde träsk and two wooden causeways.

\section{St-1658. Roma Stormyr}

$$
\text { A.D. } 1060
$$

Log from wooden causeway in the bog Roma Stormyr $\left(57^{\circ} 28^{\prime} \mathrm{N}\right.$ Lat, $18^{\circ} 24^{\prime}$ E Long).

\section{St-1677. Starrarmyr}

$1110 \pm 70$

Log from wooden causeway at church of Lärbro $\left(57^{\circ} 47^{\prime} \mathrm{N}\right.$ Lat, $18^{\circ} 48^{\prime}$ E Long). Subm. by E. Nyhlén, Gotlands Fornsalar, Visby, Gotland.

\section{St-1659. Bulverket}

$955 \pm 65$

Wood from house in great lake-dwelling "Bulverket" in Tingstäde

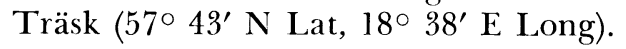

\section{Långmyrborgen series}

Peat and wood from fen castle Långmyrborgen $\left(56^{\circ} 55^{\prime} \mathrm{N}\right.$ Lat, $18^{\circ} 18^{\prime}$ E Long).

\section{St-1623. Långmyrborgen}

Sand with peat.

\section{St-1626. Långmyrborgen 24}

Wood on trample stone.

110 B.C.

St-1627. Långmyrborgen 25

$3230 \pm 120$

Peat on another stone. 
St-1629. Långmyrborgen 26

Hard peat.

St-1630. Långmyrborgen 27
$1925 \pm 70$

A.D. 25

$1790 \pm 65$

A.D. 160

Vasstäde series

Wood from palisades on NW side of fen castle in Vasstäde bog ( $57^{\circ} 12^{\prime}$ N Lat, $18^{\circ} 18^{\prime}$ E Long).

St-1562. Vasstäde A

$2110 \pm 75$

160 B.C.

St-1561. Vasstäde B $2365 \pm 70$

St-1581. Vasstäde C

415 B.c. $2270 \pm 70$

320 B.C.

\section{Halleby series I}

Charcoal from Halleby ( $58^{\circ} 42^{\prime} \mathrm{N}$ Lat, $19^{\circ} 57^{\prime} \mathrm{E}$ Long), Skärkind parish, Östergötland. Subm. by S. O. Lindqvist. This is continuation of earlier Halleby Series (Stockholm V); dated as part of large investigation of early settlements and their agriculture in this region.

St-1666. 201

$1740 \pm 70$

Uppermost charcoal layer in hearth $30 \mathrm{~cm}$ below surface.

St-1685. 202

$1740 \pm 70$

Charcoal from lowest layer among stones in same hearth as 201, 45 $\mathrm{cm}$ below surface.

St-1686. 203

$$
1355 \pm 80
$$

Uppermost charcoal layer in hearth $40 \mathrm{~cm}$ below surface.

St-1687. 204

$$
1555 \pm 70
$$

Lowest charcoal layer among stones in same hearth as 203, $60 \mathrm{~cm}$ below surface.

St-1688. 205

$$
1360 \pm 65
$$

A.D. 590

St-1689. 206

$$
1390 \pm 65
$$

St-1690. 207

A.D. 560

$1460 \pm 80$

Samples from three hearths, close together just outside fence around building lot $\mathrm{C}, 40$ to $50 \mathrm{~cm}$ below surface. 
St-1691. 208

$$
1525 \pm 70
$$

Burned remains of house construction, $10 \mathrm{~cm}$ below surface.

A.D. 425

$1790 \pm 80$

St-1692. 209

A.D. 160

St-1693. 210

$1360 \pm 75$

From hearth inside a building, $20 \mathrm{~cm}$ below surface.

A.D. 590

St-1694. 211

$1905 \pm 75$

Post hole No. 1, 20 to $30 \mathrm{~cm}$ below surface.

A.D. 45

St-1695. 212

$$
1470 \pm 90
$$

A.D. 480

\section{Halleby series II}

Subm. by Evert Baudou, Dept. of Nordic and Comparative Archeology, Univ. of Stockholm, Stockhom.

\section{St-1872. 213}

From hearth in house from Early Iron Age.

\section{St-1873. 214}

From wall in house from Early Iron Age.

\section{St-1874. 215}

From hearth in house from Early Iron Age.

St-1875. 216

From hearth under string of stones.

$$
\begin{array}{r}
1655 \pm 70 \\
\text { A.D. } 295
\end{array}
$$

$1635 \pm 75$ A.D. 315

$$
1505 \pm 70
$$

A.D. 445

$$
1430 \pm 70
$$

A.D. 520

\section{Skånings-Åsaka series}

Charcoal from culture layers at Skånings-Åsaka $\left(58^{\circ} 24^{\prime} \mathrm{N}\right.$ Lat, $14^{\circ}$ E Long), Västergötland. Subm. by Lars Thor, Dept. of History, Univ. of Stockholm. Investigation made in order to date agriculture in this area. Samples from a charcoal horizon probably resulting from intentional clearing of land by fire.

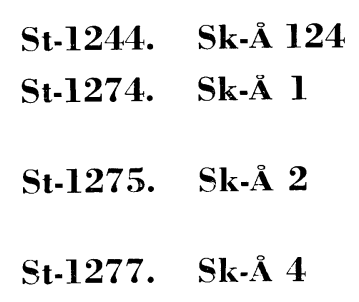

St-1244. Sk-Å 124

$330 \pm 95$ A.D. 1620

A.D. 1640

$\mathbf{3 1 0} \pm \mathbf{1 3 0}$

$355 \pm 120$

A.D. 1595 
Charcoal from a cairn or agricultural mound of stones in pine-forest area at Kilatorpet $\left(58^{\circ} 08^{\prime} \mathrm{N}\right.$ Lat, $15^{\circ} 02^{\prime} \mathrm{E}$ Long), Hardemo parish, Närke. Subm. by Siv Augustsson, Dept. of Geography, Univ. of Stockholm, Stockholm.

\section{St-1877. Ekeberga}

$385 \pm 135$

Charcoal from burnt and trampled area at Ekeberga $\left(58^{\circ} 04^{\prime} \mathrm{N}\right.$ Lat, $15^{\circ} 11^{\prime}$ E Long), Hallsbergs parish, Närke. Subm. by Siv Augustsson.

\section{Hassla series}

Charcoal from agricultural fields at Hassla $\left(58^{\circ} 26^{\prime} \mathrm{N}\right.$ Lat, $15^{\circ} 10^{\prime}$ E Long), Vallerstad parish, Östergötland. Subm. by Maud Steiner and Ulla Svennrud-Parsons, Dept. of Geography, Univ. of Stockholm, Stockholm.
St-1878. Hassla 1965:A
$<\mathbf{2 5 0}$
St-1879. Hassla 1965:B
A.D. 1365
St-1880. Hassla 1965:C

$$
2945 \pm \mathbf{3 6 0}
$$
995 B.C.

\section{Skederid series}

Charcoal from abandoned and unmapped agricultural areas in Skederid parish (59 $41^{\prime} \mathrm{N}$ Lat, $18^{\circ} 39^{\prime} \mathrm{E}$ Long), Uppland. Subm. by Ulf Sporrong, Dept. of Geography, Univ. of Stockholm.

\section{St-1883. Snesslingby I}

St-1884. Snesslingby II

St-1885. Snesslingby III

St-1886. Snesslingby IV

St-1887. Snesslingby V

St-1888. Sveden 1

St-1889. Fågla 1

St-1882. Lännaby 1

St-1881. Österlisa 1
$880 \pm 65$ A.D. 1070

$$
720 \pm 90
$$

A.D. 1230

A.D. 1580

$$
370 \pm 65
$$

A.D. 1105

A.D. 1400

$$
550 \pm 70
$$

A.D. 1075

$875 \pm 65$

$3155 \pm 65$

1205 B.C.

$1325 \pm 105$

A.D. 625

$1035 \pm 65$

A.D. 915 


\section{Alskog series}

Charcoal from Alskog parish (57 $22^{\prime} \mathrm{N}$ Lat, $18^{\circ} 39^{\prime} \mathrm{E}$ Long), Gotland. Subm. by D. Hannerberg, Dept. of Geography, Univ. of Stockholm, Stockholm.

St-1538. 64/65:2

St-1541. 64/65:4

St-1542. 64/65:5

St-1539. 6/65:7
A.D. 1350

$600 \pm 70$

$435 \pm 70$

A.D. 1515

$<250$

A.D. 1640

\section{Dysherg series}

Wood from logs in supposed building around reduction furnace for manufacture of iron at Dysberg-Dysdalen $\left(61^{\circ} 09^{\prime} \mathrm{N}\right.$ Lat, $18^{\circ} \mathrm{E}$ Long), Älvdalens parish, Dalarna. Coll. by J. P. Lamm; subm. by G. Arwidsson.

\section{St-1931. Dysberg 1}

St-1932. Dysberg 2
$705 \pm 65$

A.D. 1245

A.D. 1460

$$
490 \pm 65
$$

\section{B. Other Countries}

\section{Lerna series}

Bone from Cincinnati Univ. Excavation at Lerna $\left(37^{\circ} 36^{\prime} \mathrm{N}\right.$ Lat, $22^{\circ} 50^{\prime}$ E Long), Argolide, Greece. Subm. by N.-G. Gejvall. Dated samples are selected from collection of 2380 samples of some 25,000 animal bone fragments. Comment (N-G. G.): dates agree closely with archaeological chronology.

\section{St-1969. Lerna 1}

$3765 \pm 70$

Bones (sacrum) of domestic cattle. 1815 B.C.

\section{St-1980. Lerna 9}

Proximal part of humerus of domestic pig.

\section{Luni series}

Bone samples from Luni (42० $13^{\prime}$ Lat, $11^{\circ} 56^{\prime} \mathrm{E}$ Long), in province of Viterbo, Comune di Blera. Subm. by C. E. Östenberg, Ist. Svedese, Rome, Italy. This is continuation of Luni series in Stockholm VI (Östenberg, 1966).

St-2043. Kl 1

$4025 \pm 100$

Tibia of Bos t. dom. 
St-2042. K1 2

$3955 \pm 200$

Femur of Homo.

2005 B.C.

St-2044. K1 3

$3005 \pm 75$

Scapula of Bos t. dom.

1055 B.C.

St-2047. KI 4

$2945 \pm 80$

Radius and ulna of Bos $t$. dom.

995 B.C.

St-2045. KI 5

$3120 \pm 75$

1170 B.c.

\section{Buchy series}

Charcoal from fortification at Buchy $\left(49^{\circ} 30^{\prime} \mathrm{N}\right.$ Lat, $01^{\circ} 17^{\prime} \mathrm{E}$ Long), Normandie, France, situated on hillslope originally surrounded by two streams. It consists of a circular earthen wall and foss. Samples are collected from a furnace, probably older than the earth work, also from the main culture level. Coll. and subm. by H. Arbman, Historiska Museet, Univ. of Lund, Lund.

\section{St-1699. Buchy 1}

$920 \pm 70$

Trench Fd, 20 to $21 \mathrm{~m}$, on bottom of furnace.

St-1700. Buchy 2

A.D. 1030

Trench $\mathrm{Fd}, 19$ to $21 \mathrm{~m}$, in the furnace.

$<250$

St-1701. Buchy 3

$765 \pm 95$

Trench Fd, 20 to $21 \mathrm{~m}$, in the furnace.

A.D. 1185

\section{St-1702. Buchy 4}

$730 \pm 110$

Trench Fa, 19 to $20 \mathrm{~m}$ culture layer, with red, burnt clay, at bottom of trench.

St-1703. Buchy 5

$970 \pm 65$

Trench Fa, 18 to $20 \mathrm{~m}$, dark layer.

A.D. 980

St-1704. Buchy 6

$980 \pm 140$

Trench F, 22 to $23 \mathrm{~m}$, under the wall.

A.D. 970

St-1705. Buchy 7

$845 \pm 80$

Trench F, 13.5 to $15.5 \mathrm{~m}$, in deep pit, 3.10 below surface.

St-1706. Buchy 8

$835 \pm 65$

Trench F, $15 \mathrm{~m}$ at bottom of pit, 3.50 below surface. 


\section{Bredkälen series}

III. MISCELLANEOUS (GEOCHEMICAL) SAMPLES

Atmospheric $\mathrm{CO}_{2}$ from Bredkälen (63 $54^{\prime} \mathrm{N}$ Lat, $15^{\circ} 18^{\prime} \mathrm{E}$ Long), alt $400 \mathrm{~m}$, Jämtland, Sweden. Earlier series in Stockholm V. Subm. by Svante Odén, Internat. Meteorological Inst., Stockholm. Only selected samples have been analyzed.

$\begin{array}{ll}\text { St-2013. } & 6306 \\ \text { St-1806. } & 6307 \\ \text { St-1807. } & 6309 \\ \text { St-2017. } & 6311 \\ \text { St-1805. } & \mathbf{6 4 0 2} \\ \text { St-1802. } & \mathbf{6 4 0 5} \\ \text { St-1804. } & 6507 \\ \text { St-1803. } & 6409 \\ \text { St-2014. } & \mathbf{6 4 1 2} \\ \text { St-1801. } & 6502 \\ \text { St-2015. } & 6505 \\ \text { St-2016. } & 6508\end{array}$

$\begin{array}{cc}\delta \mathrm{C}^{13}, \% \circ & \Delta, \% \\ -\mathbf{1 7} & +\mathbf{7 8 2} \pm \mathbf{6} \\ \mathbf{- 1 2} & +\mathbf{9 1 5} \pm \mathbf{6} \\ \mathbf{- 8} & +\mathbf{9 9 3} \pm \mathbf{6} \\ \mathbf{- 1 0} & +\mathbf{8 9 6} \pm \mathbf{7} \\ -\mathbf{9} & +\mathbf{8 3 7} \pm \mathbf{6} \\ -\mathbf{9} & +\mathbf{9 0 9} \pm \mathbf{6} \\ -\mathbf{8} & +\mathbf{9 7 9} \pm \mathbf{6} \\ -\mathbf{8} & +\mathbf{9 4 0} \pm \mathbf{6} \\ \mathbf{- 1 0} & +\mathbf{8 1 4} \pm 6 \\ -\mathbf{8} & +\mathbf{7 8 5} \pm \mathbf{6} \\ \mathbf{- 1 0} & +\mathbf{7 8 6} \pm \mathbf{6} \\ \mathbf{- 1 0} & +\mathbf{7 9 3} \pm 6\end{array}$

REFERFNCFS

Date lists:
Stockholm I
Östlund, 1957
Stockholm II
Östlund, 1959
Stockholm V
Östlund, and Engstrand, 1963
Stockholm VI
Engstrand, 1965

Berger, R., Horney, A. G. and Libby, W. F., 1964, Radiocarbon dating of bone and shell from their organic components: Science, v. 144, p. 999-1001.

Berglund, B. E., 1964, The Post-Glacial shore displacement in eastern Blekinge, south-eastern Sweden; Sveriges Geologiska Undersökning, ser. C, no. 599, p. 1-47. - 1966a, Late-Quaternary vegetation in eastern Blekinge, south-eastern Sweden. A pollen-analytical study I. Late-Glacial time: Opera Botanica 12:1, p. $1-180$. Sweden. A pollen-analytical study II. Post-Glacial time: Opera Botanica 12:2, p. $1-190$.

Engstrand, L. G., 1965, Stockholm natural radiocarbon measurements VI: Radiocarbon v. 7 , p. $257-290$

Gejvall, N-G., 1960, Westerhus, medieval population and church in the light of skeletal remains: Håkan Olsson's printing office, Lund.

Hoppe, G., 1965, Submarine peat in the Shetland Islands: Geogr. Annaler, v. 47, ser. A 1965:4, p. 195-203.

Lundqvist G., 1965, $\mathrm{C}^{14}$-dateringar fran Gotland. $\mathrm{C}^{14}$-datings from Gotland (in Swedish, summary in English): Sveriges Geol. Undersökning, ser. C, no. 602.

Östenberg, C. E., 1967, Luni sul Mignone, e problemi etc. Opuscila Romana . . . , in press.

Östlund, H. G., 1957, Stockholm natural radiocarbon measurements I: Science, v. 126, p. $493-497$

1959, Stockholm natural radiocarbon measurements II: Am. Jour. Sci. Radioc. Supp., v. 1, p. 35-44.

Östlund, H. G. and Engstrand, L. G., 1963, Stockholm natural radiocarbon measurements V: Radiocarbon, v. 5, p. 203-227.

Sellstedt, H., Engstrand, L. G. and Gejvall, N.G., 1966, New application of radiocarbon dating of collagen residues in bone: Nature, v. 212, no. 5062, p. 572-574.

Svärdstedt, T., 1966, Odontological aspects of a Medieval population in the province of Jämtland/mid-Sweden/: Tiden-Barnängen printing office, in press. 